\title{
ANALYSIS OF THE ACCURACY AND CONVERGENCE OF EQUATION-FREE PROJECTION TO A SLOW MANIFOLD
}

\author{
Antonios Zagaris ${ }^{1,2}$, C. William Gear ${ }^{3,4}$, Tasso J. Kaper ${ }^{5}$ \\ And YAnnis G. KevREKIDIS ${ }^{3,6}$
}

\begin{abstract}
In [C.W. Gear, T.J. Kaper, I.G. Kevrekidis and A. Zagaris, SIAM J. Appl. Dyn. Syst. 4 (2005) 711-732], we developed a class of iterative algorithms within the context of equation-free methods to approximate low-dimensional, attracting, slow manifolds in systems of differential equations with multiple time scales. For user-specified values of a finite number of the observables, the $m$ th member of the class of algorithms $(m=0,1, \ldots)$ finds iteratively an approximation of the appropriate zero of the $(m+1)$ st time derivative of the remaining variables and uses this root to approximate the location of the point on the slow manifold corresponding to these values of the observables. This article is the first of two articles in which the accuracy and convergence of the iterative algorithms are analyzed. Here, we work directly with fast-slow systems, in which there is an explicit small parameter, $\varepsilon$, measuring the separation of time scales. We show that, for each $m=0,1, \ldots$, the fixed point of the iterative algorithm approximates the slow manifold up to and including terms of $\mathcal{O}\left(\varepsilon^{m}\right)$. Moreover, for each $m$, we identify explicitly the conditions under which the $m$ th iterative algorithm converges to this fixed point. Finally, we show that when the iteration is unstable (or converges slowly) it may be stabilized (or its convergence may be accelerated) by application of the Recursive Projection Method. Alternatively, the Newton-Krylov Generalized Minimal Residual Method may be used. In the subsequent article, we will consider the accuracy and convergence of the iterative algorithms for a broader class of systems - in which there need not be an explicit small parameter - to which the algorithms also apply.
\end{abstract}

Mathematics Subject Classification. 35B25, 35B42, 37M99, 65L20, 65P99.

Received July 10, 2008.

Published online July 8, 2009.

\section{INTRODUCTION}

The long-term dynamics of many complex chemical, physical, and biological systems simplify when a lowdimensional, attracting, invariant slow manifold is present. Such a slow manifold attracts all nearby initial

\footnotetext{
Keywords and phrases. Iterative initialization, DAEs, singular perturbations, legacy codes, inertial manifolds.

${ }^{1}$ Department of Mathematics, University of Amsterdam, Amsterdam, The Netherlands.

2 Modeling, Analysis and Simulation, Centrum Wiskunde \& Informatica, Amsterdam, The Netherlands.

${ }^{3}$ Department of Chemical Engineering, Princeton University, Princeton, NJ 08544, USA.

${ }^{4}$ NEC Laboratories USA, retired.

${ }^{5}$ Department of Mathematics and Center for BioDynamics, Boston University, Boston, MA 02215, USA. tasso@math.bu.edu

${ }^{6}$ Program in Applied and Computational Mathematics, Princeton University, Princeton, NJ 08544, USA.
} 
data exponentially, and the reduced dynamics on it govern the long term evolution of the full system. More specifically, a slow manifold is parameterized by observables which are typically slow variables or functions of variables. All nearby system trajectories decompose naturally into a fast component that contracts exponentially toward the slow manifold and a slow component which obeys the reduced system dynamics on the manifold. In this sense, the fast variables become slaved to the observables, and knowledge of the slow manifold and of the reduced dynamics on it suffices to determine the full long-term system dynamics.

The identification and approximation of slow manifolds is usually achieved by employing a reduction method. We briefly list a number of these: Intrinsic Low Dimensional Manifold (ILDM), Computational Singular Perturbation (CSP), Method of Invariant Manifold (MIM), Approximate Inertial Manifold approaches, and FraserRoussel iteration, and we refer the reader to $[6,9,20,21]$ for a more extensive listing.

\subsection{A class of iterative algorithms based on the zero-derivative principle}

In [6], we developed a class of iterative algorithms to locate slow manifolds for systems of ordinary differential equations (ODEs) of the form

$$
\begin{array}{ll}
u^{\prime}=p(u, v), & u \in \mathbf{R}^{\mathrm{N}_{\mathrm{s}}}, \\
v^{\prime}=q(u, v), & v \in \mathbf{R}^{\mathrm{N}_{\mathrm{f}}},
\end{array}
$$

where $\mathrm{N}_{\mathrm{s}}+\mathrm{N}_{\mathrm{f}} \equiv \mathrm{N}$. We treated the variables $u$ as the observables (that is, as parameterizing the slow manifold we are interested in), and we assumed that there exists an $\mathrm{N}_{\mathrm{s}}$-dimensional, attracting, invariant, slow manifold $\mathcal{L}$, which is given locally by the graph of a function $v=v(u)$. For specified values of $u$, the algorithm finds approximations to $v(u)$. However, we emphasize that we did not need explicit knowledge of which variables are fast and which are slow, only that the variables $u$ suffice to parameterize $\mathcal{L}$.

To leading order, the location of a slow manifold $\mathcal{L}$ is obtained by setting $v^{\prime}=0$, i.e., by solving $q(u, v)=0$ for $v$. Of course, the manifold defined by this equation is in general not an invariant slow manifold under the flow of the full system (1.1). It is only approximately invariant, and higher-order derivatives with respect to the (fast) time $t$ are, in general, large on it. If one requires that $v^{\prime \prime}$ vanishes, then the solutions with initial conditions at the points defined by this condition depend only on the slow time to one order higher, as $v^{\prime}$ also remains bounded in the vicinity of this manifold. Similarly, demanding that successively higher-order time derivatives vanish, we obtain manifolds where all time derivatives of lower order remain bounded. The solutions with these initial conditions depend only on the slow time to successively higher order and thus approximate, also to successively higher order, solutions on the slow manifold. In other words, demanding that time derivatives of successively higher order vanish, we filter out the fast dynamics of the solutions to successively higher orders. In this manner, the approximation of the slow manifold $\mathcal{L}$ is improved successively, as well. This idea may be traced back at least to the work of Kreiss [1,12,13], who studied systems with rapid oscillations (asymptotically large frequencies) and introduced the bounded derivative principle to find approximations of slow manifolds as the sets of points at which the derivatives are bounded (not large). The requirement here that the derivatives with respect to the (fast) time $t$ vanish (or be small) is the analog for systems (1.1) with asymptotically stable slow manifolds. A similar idea was introduced independently by Lorenz in [14], where he used a simple functional iteration scheme to approximate the zero of the first derivative, then used the converged value of this scheme to initialize a similar scheme that approximates the zero of the second derivative, and so on until successive zeroes were found to be virtually identical. See also [3,7] for other works in which a similar condition is employed.

The elements of the class of iterative algorithms introduced in [6] are indexed by $m=0,1, \ldots$ The $m$ th algorithm is designed to locate, for any fixed value of the observable $u_{0}$, an appropriate solution, $v=v_{m}\left(u_{0}\right)$, of the $(m+1)$ st derivative condition

$$
\left(\frac{\mathrm{d}^{m+1} v}{\mathrm{~d} t^{m+1}}\right)\left(u_{0}, v\right)=0 .
$$

Here, the time derivatives are evaluated along solutions of (1.1). In general, since condition (1.2) constitutes a system of $\mathrm{N}_{\mathrm{f}}$ nonlinear algebraic equations, the solution $v_{m}\left(u_{0}\right)$ cannot be computed explicitly. Also, the explicit 
form of (1.1), and thus also an analytic formula for the $(m+1)$ st time derivative in equation (1.2), may be unavailable (e.g., in equation-free or legacy code applications, see $[5,11]$ ). In this case, a numerical approximation for it has to be used. The $m$ th algorithm in the class generates an approximation $v_{m}^{\#}$ of $v_{m}\left(u_{0}\right)$, rather than $v_{m}\left(u_{0}\right)$ itself, using either an analytic formula for the time derivative or a finite difference approximation for it. In either case, the approximation $v_{m}^{\#}$ to $v_{m}\left(u_{0}\right)$ is determined through an explicit functional iteration scheme, which we now introduce.

The algorithm with general $m$ is defined by the map $\tilde{F}_{m}: \mathbf{R}^{\mathrm{N}_{\mathrm{f}}} \rightarrow \mathbf{R}^{\mathrm{N}_{\mathrm{f}}}$,

$$
\tilde{F}_{m}(v)=v-(-H)^{m+1}\left(\frac{\mathrm{d}^{m+1} v}{\mathrm{~d} t^{m+1}}\right)\left(u_{0}, v\right) .
$$

Here, $H$, which we label as the iterative step size, is an arbitrary positive number whose magnitude we fix below for stability reasons. We initialize the iteration with some value $v^{(1)}$ and generate the sequence

$$
\left\{v^{(r+1)} \equiv \tilde{F}_{m}\left(v^{(r)}\right) \mid r=1,2, \ldots\right\}
$$

In addition, one prescribes a tolerance $\mathrm{TOL}_{m}$ and terminates the iteration procedure when $\left\|v^{(r+1)}-v^{(r)}\right\|<$ $\mathrm{TOL}_{m}$ for some $r \geq 1$. The output of this $m$ th algorithm is the last member of the sequence $\left\{v^{(r+1)}\right\}$, denoted by $v_{m}^{\#}$.

As we show in this article, not only is the point $\left(u_{0}, v_{m}^{\#}\right)$ of interest for each individual $m$ because it approximates $\left(u_{0}, v\left(u_{0}\right)\right)$, but the entire sequence $\left\{\left(u_{0}, v_{m}^{\#}\right)\right\}_{m}$ is also of interest because it converges to $\left(u_{0}, v\left(u_{0}\right)\right)$ with a suitably convergent sequence $\left\{\mathrm{TOL}_{m}\right\}_{m}$. Hence, the latter point can be approximated arbitrarily well by members of that sequence, and the class of algorithms may be used as an integrated sequence of algorithms in which the output $v_{m}^{\#}$ of the $m$ th algorithm can be used to initialize the $(m+1)$ st algorithm. Of course, other initializations are also possible, and we have carried out the analysis here in a manner that is independent of which choice one makes.

Van Leemput et al. [18] employed the first $(m=0)$ algorithm in the class to initialize lattice Boltzmann models (LBM) from sets of macroscopic data in a way that eliminates the stiff dynamics triggered by a bad initialization. They showed that the algorithm they derived converges unconditionally to a fixed point close to a slow manifold, and they used the algorithm to couple a LBM to a reaction-diffusion equation along the interface with good results [19]. The algorithm has also been applied to a series of examples in [6]. It was found that the $m$ th algorithm converged exponentially for each value of $m$ that was tried, and that the accuracy of the approximation to $\left(u_{0}, v\left(u_{0}\right)\right)$ improved as the order $m$ was increased.

\subsection{Iterative algorithms based on the zero-derivative principle for explicit fast-slow-systems}

A central assumption that we made in [6] is that we work with systems (1.1) for which there exists a smooth and invertible coordinate change

$$
z=z(w) \text { with inverse } \quad w=w(z)
$$

where $w=(u, v)$ and $z=(x, y)$, which puts the system (1.1) into the explicit fast-slow form

$$
\begin{aligned}
x^{\prime} & =f(x, y, \varepsilon), & & x \in \mathbf{R}^{\mathrm{N}_{\mathrm{s}},} \\
\varepsilon y^{\prime} & =g(x, y, \varepsilon), & & y \in \mathbf{R}^{\mathrm{N}_{\mathrm{f}}} .
\end{aligned}
$$

We emphasize that, in general, we have no knowledge whatsoever of the transformation that puts system (1.1) into an explicit fast-slow form. Here, $f$ and $g$ are smooth functions of their arguments, the manifold $\mathcal{L}$ is transformed smoothly, and the matrix $\left(D_{y} g\right)_{0}(z) \equiv D_{y} g(z, 0)$ is non-singular, $\operatorname{det}\left(D_{y} g\right)_{0}(z) \neq 0$, on the manifold $\mathcal{L}_{[0]}=\{z \mid g(z, 0)=0\}$ (on which the dynamics reduce for $\varepsilon=0$ ), see also [6]. 
Due to the above assumption, it turns out to be natural to split the analysis of the accuracy and convergence of the functional iteration into two parts. In the first part, which we present in this article, we work directly on systems that are already in explicit fast-slow form (1.5). In the context of these systems, the accuracy and convergence analysis may be carried out completely in terms of the small parameter $\varepsilon$. The system geometry the slow manifold and the fast fibers transverse to $\mathcal{L}$ - makes the convergence analysis especially transparent. Then, in the second part, we work with the more general systems (1.1). For these, the accuracy analysis proceeds along similar lines as that for this first part, with the same type of result as Theorem 2.1 below. However, the convergence analysis is considerably more involved than that for explicit fast-slow systems. For these general systems, one must analyze a series of different scenarios depending on the relative orientations of (i) the tangent space to $\mathcal{L}$, (ii) the tangent spaces to the fast fibers at their base points on $\mathcal{L}$, and (iii) the hyperplane of the observables $u$. Moreover, all of the analysis must be carried out through the lens of the coordinate change (1.4) and its inverse, so that it is less transparent than it is in part one. Part two is presented in [22].

As applied specifically to explicit fast-slow systems (1.5), the $m$ th iterative algorithm (1.3) is based on the $(m+1)$ st derivative condition,

$$
\left(\frac{\mathrm{d}^{m+1} y}{\mathrm{~d} t^{m+1}}\right)\left(x_{0}, y\right)=0
$$

In particular, for each $m$ and for any arbitrary, but fixed, value of the observable $x_{0}$, one makes an initial guess for the corresponding point on $\mathcal{L}$ and uses the $m$ th iterative algorithm to approximate the appropriate zero of this $(m+1)$ st derivative, where the end (converged) result of the iteration is the improved approximation of the point on $\mathcal{L}$.

For each $m=0,1, \ldots$, the $m$ th iterative algorithm is defined by the map $F_{m}: \mathbf{R}^{\mathrm{N}_{\mathrm{f}}} \rightarrow \mathbf{R}^{\mathrm{N}_{\mathrm{f}}}$,

$$
F_{m}(y)=y-(-H)^{m+1}\left(\frac{\mathrm{d}^{m+1} y}{\mathrm{~d} t^{m+1}}\right)\left(x_{0}, y\right)
$$

where $H$ is an arbitrary positive number whose magnitude is $\mathcal{O}(\varepsilon)$ for stability reasons. We seed with some value $y^{(1)}$ and generate the sequence

$$
\left\{y^{(r+1)} \equiv F_{m}\left(y^{(r)}\right) \mid r=1,2, \ldots\right\}
$$

Here also, one prescribes a tolerance $\mathrm{TOL}_{m}$ and terminates the iteration procedure when $\left\|y^{(r+1)}-y^{(r)}\right\|<\mathrm{TOL}_{m}$ for some $r \geq 1$. The output of this $m$ th algorithm is the last member of the sequence $\left\{y^{(r+1)}\right\}$, denoted by $y_{m}^{\#}$. Finally, we note that the dependence of $F_{m}$ and $y$ on $\varepsilon$ has been suppressed in the notation to keep it compact.

\subsection{Fundamental hypotheses and essentials from Fenichel theory}

Throughout this article, we make some assumptions about the systems (1.5) and use some basic theory about slow, invariant manifolds in systems of the form (1.5). We emphasize that this theory is briefly discussed here only to provide a framework in which to analyze the algorithms; the algorithms may be applied in the much broader context of systems (1.1).

We assume that the set, $K$, in which the observables $x$ lie is compact, and that the manifold $\mathcal{L}_{[0]}$ may be expressed as the graph of a function $h_{[0]}$ for all $x \in K$. More generally, we may work with submanifolds of $\mathcal{L}_{[0]}$ if it cannot be so expressed globally. In addition, we assume that $\mathcal{L}_{[0]}$ is normally attracting under the dynamics associated with (1.5) with $\varepsilon$ set to zero, which for systems of the form (1.5) implies that the eigenvalues of the fast subsystem linearized at points on $\mathcal{L}_{[0]}$ have negative real parts. (This is a special case of normal hyperbolicity, in which none of the eigenvalues have zero real part.) Finally, in order to apply Fenichel theory [4], we assume that $\mathcal{L}_{[0]}$ is overflowing invariant at its boundary, i.e., that the vector field points either outward or tangent to the boundary at all points along it. This assumption may be satisfied by introducing a $C^{\infty}$ bump function in the vector field at the boundary of $K$, which is a perturbation of the vector field exterior to the region one is interested in, as is shown in [8]. 
Under the above assumptions, Fenichel theory $[4,8]$ gives the existence of a slow manifold $\mathcal{L}$ that is invariant with respect to the dynamics of system (1.5) when $\varepsilon$ is sufficiently small. Orbits on $\mathcal{L}$ evolve slowly, and orbits off $\mathcal{L}$ converge at a minimal exponential rate toward it and are shadowed by an orbit on it. Generally, slow manifolds are not unique; typical systems (1.5) have a family of slow manifolds, all of which are exponentially close to each other, $\mathcal{O}\left(\mathrm{e}^{-c / \varepsilon}\right)$ for some $c>0$. The choice of bump function at the boundary of $K$ selects one member of this family and makes the manifold locally unique. Moreover, due to the assumption that the unperturbed manifold $\mathcal{L}_{[0]}$ is the graph of a function $h_{[0]}$, we know that $\mathcal{L}$ is the graph of a function $h: K \rightarrow \mathbf{R}^{\mathrm{N}_{\mathrm{f}}}$,

$$
\mathcal{L}=\{(x, y) \mid x \in K, y=h(x)\} .
$$

See for example [8], Theorem 4.

The function $h$ satisfies the invariance equation

$$
g(x, h(x), \varepsilon)-\varepsilon D h(x) f(x, h(x), \varepsilon)=0,
$$

and it is $\mathcal{O}(\varepsilon)$ close to the unperturbed (a.k.a. critical) manifold uniformly for $x \in K$. It is insightful to recast this invariance equation in the form

$$
\left(-D h(x), I_{\mathrm{N}_{\mathrm{f}}}\right) G(x, h(x), \varepsilon)=0, \quad \text { where } \quad G \equiv\left(\begin{array}{c}
\varepsilon f \\
g
\end{array}\right),
$$

which admits a clear geometric interpretation. Since $\mathcal{L}$ corresponds to the zero level set of the function $-h(x)+y$ by equation (1.9), the rows of the $\mathrm{N}_{\mathrm{f}} \times \mathrm{N}$ gradient matrix $\left(-D h(x), I_{\mathrm{N}_{\mathrm{f}}}\right)$ form a basis for $\mathrm{N}_{z} \mathcal{L}$, the space normal to the slow manifold at the point $z=(x, h(x)) \in \mathcal{L}$. Thus, equation (1.11) states that the vector field $G$ is perpendicular to this space and hence contained in the space tangent to the slow manifold, $\mathrm{T}_{z} \mathcal{L}$. This is the precise meaning of invariance.

For compactness of notation, we have suppressed the dependence on $\varepsilon$ in $h(x)$. In fact, the function $h$ admits an asymptotic expansion in $\varepsilon$,

$$
h(\cdot)=\sum_{i=0} \varepsilon^{i} h_{[i]}(\cdot),
$$

where the coefficients $h_{[i]}, i=0,1, \ldots$, are determined by expanding asymptotically the left member of equation (1.10) and setting the coefficient of $\varepsilon^{i}$ equal to zero to obtain

$$
g_{i}-\sum_{\ell=0}^{i-1}\left(D h_{[\ell]}\right) f_{i-1-\ell}=0, \quad i=0,1, \ldots
$$

where the sum is understood to be empty for $i=0$. Here, $g_{i}$ and $f_{i}$ denote the coefficients of $\varepsilon^{i}$ in the asymptotic expansions of $g$ and $f$, respectively. (For asymptotic expansions of general functions $\mu(x, y, \varepsilon)$, we denote the coefficient on the term with $\varepsilon^{i}$ by $\mu_{i}(x, y)$, and we use the convention that $\mathcal{O}\left(\varepsilon^{i}\right)=\varepsilon^{i} \nu(x, y, \varepsilon)$ where $\nu$ is a bounded smooth function for all sufficiently small values of $\varepsilon$.) The first few equations are

$$
\begin{aligned}
& g_{0}=0, \\
& \left(D_{y} g\right)_{0} h_{[1]}+\left(D_{\varepsilon} g\right)_{0}-\left(D h_{[0]}\right) f_{0}=0 .
\end{aligned}
$$

Here, equation (1.13) is satisfied identically, equation (1.14) yields the coefficient $h_{[1]}$, and so on.

The ILDM method of [15] employs a geometric condition that is similar to equation (1.11). In particular, the ILDM method uses a Schur decomposition of the vector field into block triangular fast-slow form and then approximates the true invariant slow manifold by the set of points at which the vector field $G$ is orthogonal to the orthogonal complement of the slow subspace of the Jacobian. As a result, the ILDM condition resembles equation (1.11), but with only information about the linearized vector field, whereas equation (1.11) contains all of the nonlinear terms from the invariance equation. See also [9]. 


\subsection{Statement of the main results}

In this article, we first examine the $m$ th iterative algorithm in which an analytical formula for the $(m+1)$ st derivative is used, and we prove that it has a fixed point $y=h_{m}\left(x_{0}\right)$, which is $\mathcal{O}\left(\varepsilon^{m+1}\right)$ close to the corresponding point $h\left(x_{0}\right)$ on the invariant manifold $\mathcal{L}$, for each $m=0,1, \ldots$ See Theorem 2.1 below.

Second, we determine the conditions on $\left(D_{y} g\right)_{0}$, which is the leading order term in the fast part of the Jacobian, under which the $m$ th iterative algorithm converges to this fixed point, again with an analytical formula for the $(m+1)$ st derivative. The normal attractivity of the manifold $\mathcal{L}_{[0]}$ plays a central role in making this determination possible. In particular, for $m=0$, the iteration converges for all systems (1.5) for which $\left(D_{y} g\right)_{0}$ is uniformly Hurwitz on $\mathcal{L}_{[0]}$ and provided that the iterative step size $H$ is small enough. For each $m \geq 1$, convergence of the algorithm imposes more stringent conditions on $H$ and on the spectrum of $\left(D_{y} g\right)_{0}$. We denote this spectrum by $\sigma\left(\left(D_{y} g\right)_{0}\right)$. In particular, if $\sigma\left(\left(D_{y} g\right)_{0}\right)$ is contained in certain sets in the complex plane, which we identify completely, then the iteration converges for small enough values of the iterative step size $H$, see Theorem 3.1. These sets do not cover the entire half-plane, and thus complex eigenvalues can, in general, make the algorithm divergent.

Third, we show explicitly how the Recursive Projection Method (RPM) of Shroff and Keller [17] stabilizes the functional iteration for each $m \geq 1$ in those regimes where the iteration is unstable. This stabilization result is useful for practical implementation in the equation-free context; and, the RPM may also be used to accelerate convergence in those regimes in which the iterations converge slowly. Alternatively, the Newton-Krylov Generalized Minimal Residual Method (NK-GMRES [10]) may be used to achieve this stabilization.

Fourth, we analyze the influence of the tolerance, or stopping criterion, used to terminate the functional iteration. We show that, when the tolerance $\mathrm{TOL}_{m}$ for the $m$ th algorithm is set to $\mathcal{O}\left(\varepsilon^{m+1}\right)$, the output $y_{m}^{\#}$ also satisfies the asymptotic estimate $\left\|y_{m}^{\#}-h\left(x_{0}\right)\right\|=\mathcal{O}\left(\varepsilon^{m+1}\right)$.

Finally, we extend the accuracy and convergence analyses to the case where a forward difference approximation of the $(m+1)$ st derivative is used in the iteration, instead of the analytical formula. As to the accuracy, we find that the $m$ th iterative algorithm also has a fixed point $y=\hat{h}_{m}\left(x_{0}\right)$ which is $\mathcal{O}\left(\varepsilon^{m+1}\right)$ close to $h\left(x_{0}\right)$, so that the iteration in this case is as accurate asymptotically as the iteration with the analytical formula. Then, as to the stability, we find that the $m$ th iterative algorithm with a forward difference approximation of the $(m+1)$ st derivative converges unconditionally for $m=0$. Moreover, for $m=1,2, \ldots$, the convergence is for a continuum of values of the iterative step size $H$ and without further restrictions on $\left(D_{y} g\right)_{0}$, other than that it is uniformly Hurwitz on $\mathcal{L}_{[0]}$, see Theorem 6.1. These advantages stem from the use of a forward difference approximation, and we will show in a future work that the use of implicitly defined maps $F_{m}$ yields similar advantages.

We remark that the condition imposed at the edge of the domain $K$ in the numerical implementation determines which of the particular slow manifolds out of the family of manifolds is the one obtained. Of course, since they are all $\mathcal{O}\left(\mathrm{e}^{-c / \varepsilon}\right)$ close for some $c>0$, the asymptotic expansions are all the same to all orders in $\varepsilon$. Hence, this particular detail of the numerical implementation does not alter the asymptotic accuracy of the results.

\section{EXISTENCE OF A FIXED POINT $h_{m}\left(x_{0}\right)$ AND ITS PROXimity to $h\left(x_{0}\right)$}

We rewrite the map $F_{m}$, given in equation (1.7), as

$$
F_{m}(y)=y-L_{m}\left(x_{0}, y\right)
$$

where the function $L_{m}: \mathbf{R}^{\mathrm{N}} \rightarrow \mathbf{R}^{\mathrm{N}_{\mathrm{f}}}$ is given by

$$
L_{m}(z) \equiv(-H)^{m+1}\left(\frac{\mathrm{d}^{m+1} y}{\mathrm{~d} t^{m+1}}\right)(z), \quad \text { for any } m=0,1, \ldots
$$


where $z=\left(x_{0}, y\right)$. The fixed points, $y=h_{m}\left(x_{0}\right)$, of $F_{m}$ are determined by the equation

$$
L_{m}\left(x_{0}, h_{m}\left(x_{0}\right)\right)=0
$$

that is, by the $(m+1)$ st derivative condition (1.6). The desired results on the existence of the fixed point $h_{m}\left(x_{0}\right)$ and on its proximity to $h\left(x_{0}\right)$ are then immediately at hand from the following theorem:

Theorem 2.1. For each $m=0,1, \ldots$ there is an $\varepsilon_{m}>0$ such that, for $0<\varepsilon \leq \varepsilon_{m}$, the $(m+1)$ st derivative condition (1.6),

$$
L_{m}(x, y) \equiv(-H)^{m+1}\left(\frac{\mathrm{d}^{m+1} y}{\mathrm{~d} t^{m+1}}\right)(x, y)=0,
$$

can be solved uniquely for $y$ to yield an $\mathrm{N}_{\mathrm{s}}$-dimensional manifold $\mathcal{L}_{m}$ which is the graph of a function $h_{m}: K \rightarrow$ $\mathbf{R}^{\mathrm{N}_{\mathrm{f}}}$ over $x$. Moreover, the asymptotic expansions of $h_{m}$ and $h$ agree up to and including terms of $\mathcal{O}\left(\varepsilon^{m}\right)$,

$$
h_{m}(\cdot)=\sum_{i=0} \varepsilon^{i} h_{m, i}(\cdot)=\sum_{i=1}^{m} \varepsilon^{i} h_{[i]}(\cdot)+\mathcal{O}\left(\varepsilon^{m+1}\right) .
$$

This theorem guarantees that, for each $x_{0} \in K$, there exists an isolated fixed point $y=h_{m}\left(x_{0}\right)$ of the functional iteration algorithm. Moreover, this fixed point varies smoothly with $x_{0}$, and the approximation $\left(x_{0}, h_{m}\left(x_{0}\right)\right)$ of the point $\left(x_{0}, h\left(x_{0}\right)\right)$ on the actual invariant slow manifold is valid up to $\mathcal{O}\left(\varepsilon^{m+1}\right)$.

The remainder of this section is devoted to the proof of this theorem. We prove it for $m=0$ and $m=1$ in Sections 2.1 and 2.2, respectively. Then, in Section 2.3, we use induction to prove the theorem for general $m$.

\subsection{Proof of Theorem 2.1 for $\boldsymbol{m}=\mathbf{0}$}

We show, for each $x \in K$, that $L_{0}(z)$ has a root $y=h_{0}(x)$, that $h_{0}$ lies $\mathcal{O}(\varepsilon)$ close to $h_{[0]}(x)$ (recall that $\left.\mathcal{L}_{[0]}=\operatorname{graph}\left(h_{[0]}\right)\right)$, the corresponding point on the critical manifold, and that the graph of the function $h_{0}$ over $K$ forms a manifold.

For $m=0$, definition (2.2), the chain rule, and the ODEs (1.5) yield

$$
L_{0}=-H y^{\prime}=-\varepsilon^{-1} H g
$$

Substituting the asymptotic expansion $y=h_{0}(x)=\sum_{i=0} \varepsilon^{i} h_{0, i}(x)$ into this formula and combining it with the condition $L_{0}=0$, we find that, to leading order,

$$
g\left(x, h_{0,0}(x), 0\right)=0,
$$

where we have removed the $\mathcal{O}(1)$, nonzero, scalar quantity $-H / \varepsilon$. In comparison, the invariance equation (1.10) yields

$$
g\left(x, h_{[0]}(x), 0\right)=0,
$$

to leading order, see equation (1.13). Thus $h_{0,0}$ can be chosen to be equal to $h_{[0]}$, and $L_{0}(z)$ has a root that is $\mathcal{O}(\varepsilon)$ close to $y=h(x)$.

It remains to show that the graph of the function $h_{0}$ is an $\mathrm{N}_{\mathrm{s}}$-dimensional manifold $\mathcal{L}_{0}$. Using equation (2.4), we calculate

$$
\left(D_{y} L_{0}\right)=-\varepsilon^{-1} H\left(D_{y} g\right),
$$

where all quantities are evaluated at $\left(x, h_{0}(x), \varepsilon\right)$. Moreover,

$$
\left(D_{y} L_{0}\right)\left(x, h_{0}(x)\right)=-\varepsilon^{-1} H\left(D_{y} g\right)_{0}+\mathcal{O}(\varepsilon)
$$

with $\left(D_{y} g\right)_{0}=\left(D_{y} g\right)\left(x, h_{0,0}(x), 0\right)=\left(D_{y} g\right)\left(x, h_{[0]}(x), 0\right)$, since $h_{0,0}=h_{[0]}$. Thus,

$$
\operatorname{det}\left(D_{y} L_{0}\right)\left(x, h_{0}(x)\right) \neq 0, \quad \text { for all } x \in K,
$$


because $H=\mathcal{O}(\varepsilon)$ by assumption and because $\operatorname{det}\left(D_{y} g\right)_{0} \neq 0$, see the Introduction. Therefore, $\mathcal{L}_{0}$ is an $\mathrm{N}_{\mathrm{s}}$-dimensional manifold by the Implicit Function Theorem and [16], Theorem 1.13. This completes the proof of the theorem for the case $m=0$.

\subsection{The proof of Theorem 2.1 for $m=1$}

In this section, we treat the $m=1$ case. Technically speaking, one may proceed directly from the $m=0$ case to the induction step for general $m$. Nevertheless, we find it useful to present a concrete instance and a preview of the general case, and hence we give a brief analysis of the $m=1$ case here.

We calculate

$$
L_{1}=(-H)^{2} y^{\prime \prime}=-H\left(-H y^{\prime}\right)^{\prime}=-H L_{0}^{\prime}=-\varepsilon^{-1} H\left(D_{z} L_{0}\right) G .
$$

Using the ODEs (1.5) and equation (2.4), we rewrite this as

$$
L_{1}=\left(-\varepsilon^{-1} H\right)^{2}\left[\varepsilon\left(D_{x} g\right) f+\left(D_{y} g\right) g\right] .
$$

We recall that the solution is denoted by $y=h_{1}(x)$ and that we write its asymptotic expansion as $h_{1}(x)=$ $\sum_{i=0} \varepsilon^{i} h_{1, i}(x)$. Substituting this expansion into equation (2.6) and recalling that $H=\mathcal{O}(\varepsilon)$, we obtain at $\mathcal{O}(1)$

$$
L_{1}=\left(-\varepsilon^{-1} H\right)^{2}\left[\left(D_{y} g\right)_{0} g_{0}+\mathcal{O}(\varepsilon)\right]
$$

where $\left(D_{y} g\right)_{0}=\left(D_{y} g\right)\left(x, h_{1,0}(x), 0\right)$. Hence, $y=h_{[0]}(x)$ is a root of $L_{1}$ to leading order by equation $(2.5)$. Also, since by assumption we have that $\operatorname{det}\left(D_{y} g\right)_{0} \neq 0$, we know that the root is locally unique and hence that $h_{1,0}=h_{[0]}$.

At $\mathcal{O}(\varepsilon)$, we obtain

$$
\left(-\varepsilon^{-1} H\right)^{2}\left(D_{y} g\right)_{0}\left[\left(D_{y} g\right)_{0}^{-1}\left(D_{x} g\right)_{0} f_{0}+\left(D_{y} g\right)_{0} h_{1,1}+\left(D_{\varepsilon} g\right)_{0}\right]=0,
$$

where we used the expansion

$$
g\left(\cdot, h_{1}(\cdot), \varepsilon\right)=g_{0}+\varepsilon\left[\left(D_{y} g\right)_{0} h_{1,1}+\left(D_{\varepsilon} g\right)_{0}\right]+\mathcal{O}\left(\varepsilon^{2}\right)
$$

and that $g_{0}(\cdot)=g\left(\cdot, h_{1,0}(\cdot), 0\right)=g\left(\cdot, h_{[0]}(\cdot), 0\right)=0$. Differentiating both members of the identity $g\left(\cdot, h_{[0]}(\cdot), 0\right)=$ 0 with respect to the argument, we obtain

$$
\left(D_{x} g\right)_{0}+\left(D_{y} g\right)_{0} D h_{[0]}=0
$$

whence $\left(D_{y} g\right)_{0}^{-1}\left(D_{x} g\right)_{0}=-D h_{[0]}$. Removing also the invertible prefactor $(-H / \varepsilon)^{2}\left(D_{y} g\right)_{0}$, we find that equation (2.7) becomes

$$
-\left(D h_{[0]}\right) f_{0}+\left(D_{y} g\right)_{0} h_{1,1}+\left(D_{\varepsilon} g\right)_{0}=0 .
$$

This equation is identical to equation (1.14), and thus $h_{1,1}=h_{[1]}$. Hence, we have shown that the asymptotic expansion of $h_{1}(x)$ agrees with that of $h(x)$ up to and including terms of $\mathcal{O}(\varepsilon)$, as claimed for $m=1$.

Finally, the graph of the function $h_{1}$ forms an $\mathrm{N}_{\mathrm{s}}$-dimensional manifold $\mathcal{L}_{1}$. This may be shown in a manner similar to that used above for $\mathcal{L}_{0}$ in the case $m=0$. This completes the proof for $m=1$.

\subsection{The induction step: the proof of Theorem 2.1 for general $m$}

In this section, we carry out the induction step that establishes Theorem 2.1 for all $m$. We assume that the conclusion of Theorem 2.1 is true for $m$ and show that it also holds for $m+1$, i.e., that the condition

$$
\left[\left(D_{z} L_{m}\right)(x, y)\right] G(x, y, \varepsilon)=0
$$


can be solved uniquely for $y$ to yield $y=h_{m+1}(x)$, where

$$
h_{m+1}(\cdot)=\sum_{i=0}^{m+1} \varepsilon^{i} h_{[i]}(\cdot)+\mathcal{O}\left(\varepsilon^{m+2}\right)
$$

To begin with, we recast the $(m+1)$ st derivative condition equation $(2.3)$ in a form that is reminiscent of the invariance equation, equation (1.11). Let $m \geq 0$ be arbitrary but fixed. It follows from definition (2.2), equation (1.11), and equation (1.5) that

$$
L_{m}=-H \frac{\mathrm{d}}{\mathrm{d} t}\left((-H)^{m} \frac{\mathrm{d}^{m} y}{\mathrm{~d} t^{m}}\right)=-H \frac{\mathrm{d} L_{m-1}}{\mathrm{~d} t}=-\varepsilon^{-1} H\left(D_{z} L_{m-1}\right) G .
$$

Therefore, the $(m+1)$ st derivative condition $(2.3)$ can be rewritten in the desired form as

$$
\left(D_{z} L_{m-1}\right) G=0
$$

where we have removed the $\mathcal{O}(1)$, nonzero, scalar quantity $-H / \varepsilon$.

The induction step will now be established using a bootstrapping approach. First, we consider a modified version of equation (2.8), namely the condition

$$
\left[\left(D_{z} L_{m}\right)\left(x, h_{m}(x)\right)\right] G(x, y, \varepsilon)=0
$$

in which the matrix $D_{z} L_{m}$ is evaluated on $\mathcal{L}_{m}$ (already determined at the $m$ th iteration) instead of on the as-yet unknown $\mathcal{L}_{m+1}$. This equation is easier to solve for the unknown $y$, since $y$ appears only in $G$. We now show that the solution $y=\tilde{h}_{m+1}(x)$ of this condition approximates $h$ up to and including $\mathcal{O}\left(\varepsilon^{m+1}\right)$ terms.

Lemma 2.1. For $\varepsilon>0$ sufficiently small, the condition (2.11) can be solved uniquely for y to yield

$$
y=\tilde{h}_{m+1}(x)=\sum_{i=0}^{m+1} \varepsilon^{i} h_{[i]}(x)+\mathcal{O}\left(\varepsilon^{m+2}\right), \quad \text { for all } x \in K
$$

Now, with this first lemma in hand, we bootstrap up from the solution $y=\tilde{h}_{m+1}$ of this modified condition to find the solution $y=h_{m+1}$ of the full $(m+1)$ st derivative condition, equation (2.8). Specifically, we show that their asymptotic expansions agree up to and including terms of $\mathcal{O}\left(\varepsilon^{m+1}\right)$,

Lemma 2.2. For $\varepsilon>0$ sufficiently small, the condition (2.8) can be solved uniquely for y to yield

$$
y=h_{m+1}(x)=\sum_{i=0}^{m+1} \varepsilon^{i} \tilde{h}_{m+1, i}(x)+\mathcal{O}\left(\varepsilon^{m+2}\right), \quad \text { for all } x \in K .
$$

These lemmata are proven in Appendix 7, and Theorem 2.1 follows directly from them.

\section{Stability analysis of The Fixed POint $h_{m}\left(x_{0}\right)$}

In this section, we analyze the stability type of the fixed point $y=h_{m}\left(x_{0}\right)$ of the functional iteration scheme given by $F_{m}(y)$. To fix the notation, we let

$$
\sigma\left(D_{y} g\right)_{0}=\left\{\lambda_{k}=\lambda_{k, R}+\mathrm{i} \lambda_{k, I}=\left|\lambda_{k}\right| \mathrm{e}^{\mathrm{i} \theta_{k}}=\lambda_{k, R}\left(1+\mathrm{i} \tan \theta_{k}\right): k=1, \ldots, \mathrm{N}_{\mathrm{f}}\right\}
$$



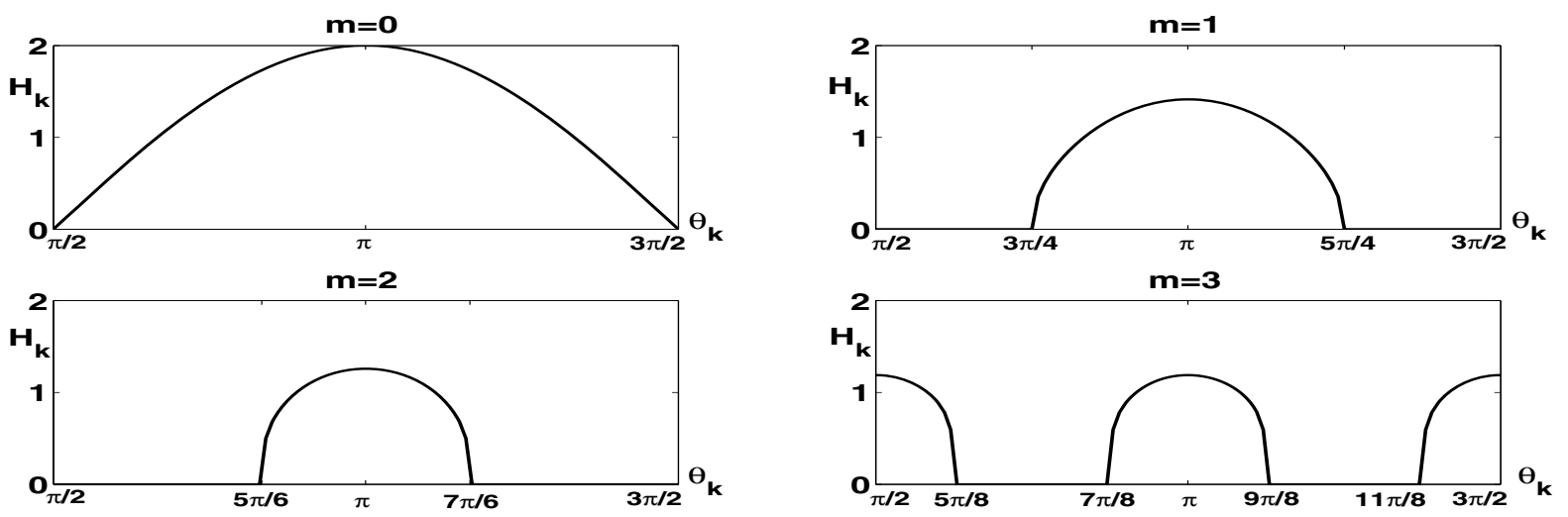

Figure 1. $H_{k}^{\max }$ as a function of $\theta_{k} \in(\pi / 2,3 \pi / 2)$, for $m=0,1,2,3$. The eigenvalue $\mu_{k}$ is stable for all $0<H_{k}<H_{k}^{\max }$.

and remark that normal attractivity of the slow manifold implies that $\lambda_{k, R}<0$ (equivalently, $\pi / 2<\theta_{k}<3 \pi / 2$ ) for all $k=1, \ldots, \mathrm{N}_{\mathrm{f}}$. Then, we prove the following theorem:

Theorem 3.1. For each $m=0,1, \ldots$ there is an $\varepsilon_{m}>0$ such that, for $0<\varepsilon \leq \varepsilon_{m}$, the functional iteration scheme defined by $F_{m}$ is stable if and only if the following two conditions are satisfied for all $k=1, \ldots, \mathrm{N}_{\mathrm{f}}$ :

$$
\theta_{k} \in \mathcal{S}_{m} \equiv \bigcup_{j=0, \ldots, m}\left(\frac{2 m+4 j+1}{2(m+1)} \pi, \frac{2 m+4 j+3}{2(m+1)} \pi\right) \cap\left[\left(\frac{\pi}{2}, \frac{3 \pi}{2}\right) \bmod 2 \pi\right]
$$

and

$$
0<H_{k}<H_{k}^{\max } \equiv\left[2 \cos \left((m+1)\left(\theta_{k}-\pi\right)\right)\right]^{1 /(m+1)}, \quad \text { where } \quad H_{k} \equiv \frac{\left|\lambda_{k}\right|}{\varepsilon} H .
$$

In particular, if $\lambda_{1}, \ldots, \lambda_{\mathrm{N}_{\mathrm{f}}}$ are real, then the functional iteration is stable for all $H$ satisfying

$$
H<H^{\max } \equiv 2^{1 /(m+1)} \frac{\varepsilon}{\left\|D_{y} g\right\|_{2}}
$$

The graphs of the stability regions for $m=0,1,2,3$ are given in Figure 1.

We now prove this theorem. By definition, $h_{m}\left(x_{0}\right)$ is exponentially attracting if and only if

$$
\sigma\left(\left(D F_{m}\right)\left(h_{m}\left(x_{0}\right)\right)\right) \subset \mathrm{B}(0 ; 1)
$$

where $\mathrm{B}(0 ; 1)$ denotes the open ball of radius one centered at the origin. To determine the spectrum of $\left(D F_{m}\right)\left(h_{m}\left(x_{0}\right)\right)$, we use equation $(2.1)$ and Lemma B.1 to obtain

$$
\begin{aligned}
\left(D F_{m}\right)(y) & =I_{\mathrm{N}_{\mathrm{f}}}-\left(D_{y} L_{m}\right)\left(x_{0}, y\right) \\
& =I_{\mathrm{N}_{\mathrm{f}}}-\left(-\varepsilon^{-1} H\left(D_{y} g\right)\left(x_{0}, y, 0\right)\right)^{m+1}+\mathcal{O}(\varepsilon)+\mathcal{O}\left(\left\|g_{0}\left(x_{0}, y\right)\right\|\right) .
\end{aligned}
$$

Letting $y=h_{m}\left(x_{0}\right)$ in this expression and observing that $\left\|g_{0}\left(x_{0}, h_{m}\left(x_{0}\right)\right)\right\|=\mathcal{O}(\varepsilon)$ by virtue of the estimate $h_{m}=h_{[0]}+\mathcal{O}(\varepsilon)$ (see Thm. 2.1) and equation (2.5), we obtain to leading order

$$
\left(D F_{m}\right)\left(h_{m}\left(x_{0}\right)\right)=I_{\mathrm{N}_{\mathrm{f}}}-\left(-\varepsilon^{-1} H D_{y} g\right)_{0}^{m+1}
$$


where $z_{m}=\left(x_{0}, h_{m}\left(x_{0}\right)\right)$ and the notation $(\cdot)_{0}$ signifies that the quantity in parentheses is evaluated at the point $\left(x_{0}, h_{[0]}\left(x_{0}\right)\right) \in \mathcal{L}_{[0]}$. Recalling the definition $H_{k}=\left|\lambda_{k}\right| H / \varepsilon$, we find to leading order

$$
\sigma\left(\left(D F_{m}\right)\left(h_{m}\left(x_{0}\right)\right)\right)=\left\{\mu_{k}=1-H_{k}^{m+1} \mathrm{e}^{i(m+1)\left(\theta_{k}-\pi\right)} \mid k=1, \ldots, \mathrm{N}_{\mathrm{f}}\right\} .
$$

In view of equation (3.7), condition (3.5) becomes

$$
\left|1-H_{k}^{m+1} \mathrm{e}^{\mathrm{i}(m+1)\left(\theta_{k}-\pi\right)}\right|<1, \quad \text { for all } k=1, \ldots, \mathrm{N}_{\mathrm{f}} .
$$

Here, we note that higher order terms omitted from formula (3.7) do not affect stability for small enough values of $\varepsilon$, because the stability region $\mathrm{B}(0 ; 1)$ is an open set. Next, we study the circumstances in which this stability condition is satisfied. This study naturally splits into the following two cases:

Case 1. The eigenvalues $\lambda_{1}, \ldots, \lambda_{\mathrm{N}_{\mathrm{f}}}$ are real. This is the case, for example, when the fast part of system (1.5) corresponds to a spatial discretization of a self-adjoint operator. Here, $\theta_{k}=\pi$ for all $k$, and thus condition (3.8) reduces to

$$
0<H_{k}^{m+1}<2, \quad \text { for all } k=1, \ldots, \mathrm{N}_{\mathrm{f}} \text { and where } H_{k}=\left|\lambda_{k}\right| \varepsilon^{-1} H,
$$

which is satisfied if equation (3.4) holds.

Case 2. Some of the eigenvalues $\lambda_{1}, \ldots, \lambda_{\mathrm{N}_{\mathrm{f}}}$ have nonzero imaginary parts. Using equation (3.7), we calculate

$$
\left|\mu_{k}\right|^{2}=1+H_{k}^{m+1}\left[H_{k}^{m+1}-2 \cos \left((m+1)\left(\theta_{k}-\pi\right)\right)\right]
$$

This equation shows that $\left|\mu_{k}\right|^{2}$ is a convex quadratic function of $H_{k}^{m+1}$ with $\left|\mu_{k}\right|^{2}=1$ for $H_{k}=0$. Convexity implies that, if there exists a second, positive solution $H_{k}^{\max }$ to the equation $\left|\mu_{k}\right|^{2}=1$, then $\left|\mu_{k}\right|<1$ for all $0<H_{k}<H_{k}^{\max }$. Plainly, $\left|\mu_{k}\right|=1$ implies, for $H \neq 0$,

$$
\left(H_{k}^{\max }\right)^{m+1}-2 \cos \left((m+1)\left(\theta_{k}-\pi\right)\right)=0
$$

which yields condition (3.3). Further, the condition that $H_{1}^{\max }, \ldots, H_{\mathrm{N}_{\mathrm{f}}}^{\max }$ be real and positive translates into condition (3.2). This completes the proof of Theorem 3.1.

It is useful to write formula (3.3) explicitly for the first several values of $m$. For $m=0$, formula (3.3) becomes

$$
H_{k}^{\max }=-2 \cos \theta_{k}
$$

see Figure 1. We note that $H_{k}^{\max }>0$ for all $\theta_{k} \in \mathcal{S}_{0}=(\pi / 2,3 \pi / 2)$, and thus the fixed point $h_{0}\left(x_{0}\right)$ is stable for all $0<H<H^{\max }$, where $H^{\max }=\varepsilon \min _{k}\left(\left|\lambda_{k}\right|^{-1} H_{k}^{\max }\right)$.

For $m=1$, formula (3.3) becomes

$$
H_{k}^{\max }=\sqrt{2 \cos \left(2 \theta_{k}\right)}
$$

see Figure 1. We see that, on $(\pi / 2,3 \pi / 2), H_{k}^{\max }>0$ only if $\theta_{k}$ lies in the subinterval $\mathcal{S}_{1}=(3 \pi / 4,5 \pi / 4)$. Therefore, the fixed point $h_{1}\left(x_{0}\right)$ is stable if and only if (i) $\theta_{k} \in \mathcal{S}_{1}$, for all $k=1, \ldots, \mathrm{N}_{\mathrm{f}}$, and (ii) $0<H<$ $H^{\max }=\varepsilon \min _{k}\left(\left|\lambda_{k}\right|^{-1} H_{k}^{\max }\right)$.

For $m=2$, formula (3.3) becomes

$$
H_{k}^{\max }=-\left[2 \cos \left(3 \theta_{k}\right)\right]^{1 / 3}
$$

see Figure 1. Here also, $H_{k}^{\max }>0$ on $(\pi / 2,3 \pi / 2)$ only if $\theta_{k}$ lies in the subinterval $\mathcal{S}_{2}=(5 \pi / 6,7 \pi / 6)$. Thus, $h_{2}\left(x_{0}\right)$ is stable if and only if (i) $\theta_{k} \in \mathcal{S}_{2}$, for all $k=1, \ldots, \mathrm{N}_{\mathrm{f}}$, and (ii) $0<H<H^{\max }=\varepsilon \min _{k}\left(\left|\lambda_{k}\right|^{-1} H_{k}^{\max }\right.$ ).

For $m=3$, formula (3.3) becomes

$$
H_{k}^{\max }=\left[2 \cos \left(4 \theta_{k}\right)\right]^{1 / 4},
$$


see Figure 1. We observe that, on $(\pi / 2,3 \pi / 2), H_{k}^{\max }>0$ only if $\theta_{k}$ lies in the subdomain $\mathcal{S}_{3}=(\pi / 2,5 \pi / 8) \cup$ $(7 \pi / 8,9 \pi / 8) \cup(11 \pi / 8,3 \pi / 2)$. Therefore, the fixed point $h_{3}\left(x_{0}\right)$ is stable if and only if (i) $\theta_{k} \in \mathcal{S}_{3}$, for all $k=1, \ldots, \mathrm{N}_{\mathrm{f}}$, and (ii) $0<H<H^{\max }=\varepsilon \min _{k}\left(\left|\lambda_{k}\right|^{-1} H_{k}^{\max }\right)$.

\section{Stabilization of the Algorithm Using RPM}

In the previous section, we saw that, for any $m \geq 1$, the $m$ th algorithm in our class of algorithms may have a number of eigenvalues that either are unstable or have modulus only slightly less than one. In this section, we demonstrate how the recursive projection method (RPM) of Shroff and Keller [17] may be used to stabilize the algorithm or to accelerate its convergence in all such cases.

For the sake of clarity, we assume that $\left(D F_{m}\right)\left(h_{m}\left(x_{0}\right)\right)$ has $M$ eigenvalues, labeled $\left\{\mu_{1}, \ldots, \mu_{M}\right\}$, that lie outside the disk $\mathrm{B}(0 ; 1-\delta)$, for some small, user-specified $\delta>0$, and that the remaining $\mathrm{N}_{\mathrm{f}}-M$ eigenvalues $\left\{\mu_{M+1}, \ldots, \mu_{\mathrm{N}_{\mathrm{f}}}\right\}$ lie inside it. We let $\mathbb{P}$ denote the maximal invariant subspace of $\left(D F_{m}\right)\left(h_{m}\left(x_{0}\right)\right)$ corresponding to $\left\{\mu_{1}, \ldots, \mu_{M}\right\}$ and $P$ denote the orthogonal projection operator from $\mathbf{R}^{\mathrm{N}_{\mathrm{f}}}$ onto that subspace. Additionally, we use $\mathbb{Q}$ to denote the orthogonal complement of $\mathbb{P}$ in $\mathbf{R}^{\mathrm{N}_{\mathrm{f}}}$ and $Q=I_{\mathrm{N}_{\mathrm{f}}}-P$ to denote the associated orthogonal projection operator. These definitions induce an orthogonal decomposition of $\mathbf{R}^{\mathrm{N}_{\mathrm{f}}}$,

$$
\mathbf{R}^{\mathrm{N}_{\mathrm{f}}}=\mathbb{P} \oplus \mathbb{Q}=P \mathbf{R}^{\mathrm{N}_{\mathrm{f}}} \oplus Q \mathbf{R}^{\mathrm{N}_{\mathrm{f}}}
$$

and, as a result, each $y \in \mathbf{R}^{\mathrm{N}_{f}}$ has a unique decomposition $y=\tilde{p}+\tilde{q}$, with $\tilde{p}=P y \in \mathbb{P}$ and $\tilde{q}=Q y \in \mathbb{Q}$. The fixed point problem $y=F_{m}(y)$ may now be written as

$$
\begin{aligned}
& \tilde{p}=P F_{m}(\tilde{p}+\tilde{q}), \\
& \tilde{q}=Q F_{m}(\tilde{p}+\tilde{q}) .
\end{aligned}
$$

The fundamental idea of RPM is to use Newton iteration on equation (4.1) and functional iteration on equation (4.2). In particular, we decompose the point $y^{(1)}$ (which was used to generate the sequence $\left\{y^{(r+1)}\right\}$ in equation (1.8)) via

$$
y^{(1)}=\tilde{p}^{(1)}+\tilde{q}^{(1)}=P y^{(1)}+Q y^{(1)} .
$$

Then, we apply Newton iteration on equation (4.1) (starting with $\tilde{p}^{(1)}$ ) and functional iteration on equation (4.2) (starting with $\left.\tilde{q}^{(1)}\right)$,

$$
\begin{aligned}
& \tilde{p}^{(r+1)}=\tilde{p}^{(r)}+\left[I_{M}-P\left(D F_{m}\left(\tilde{p}^{(r)}+\tilde{q}^{(r)}\right)\right) P\right]^{-1} P F_{m}\left(\tilde{p}^{(r)}+\tilde{q}^{(r)}\right) \\
& \tilde{q}^{(r+1)}=Q F_{m}\left(\tilde{p}^{(r)}+\tilde{q}^{(r)}\right) .
\end{aligned}
$$

The iteration is terminated when $\left\|y^{(r+1)}-y^{(r)}\right\|<\mathrm{TOL}_{m}$, for some $r \geq 1$, as was also the case with functional iteration.

Application of Theorem 3.13 from [17] directly yields that the stabilized (or accelerated) iterative scheme (4.3) converges for all initial guesses $y^{(1)}$ close enough to the fixed point $h_{m}\left(x_{0}\right)$, as long as

$$
1 \notin \sigma\left(P\left(D F_{m}\left(h_{m}\left(x_{0}\right)\right)\right) P\right)=\left\{\mu_{1}, \ldots, \mu_{M}\right\}
$$

In our case, this condition is satisfied for all $H>0$, because the fact that $\mathcal{L}$ is normally attracting implies that each eigenvalue $\lambda_{k}$ of $D_{y} g$ is bounded away from zero uniformly over the domain $K$ on which the slow manifold is defined. Thus, the iteration scheme (4.3) converges. 


\section{TUNing OF THE TOLERANCE}

In this section, we establish that, for every $m=0,1, \ldots,\left\|y_{m}^{\#}-h\left(x_{0}\right)\right\|=\mathcal{O}\left(\varepsilon^{m+1}\right)$ whenever $\mathrm{TOL}_{m}=$ $\mathcal{O}\left(\varepsilon^{m+1}\right)$. The value returned by the functional iteration is within the tolerance of the point on the true slow manifold for sufficiently small values of the tolerance.

The brunt of the analysis needed to prove this principal result involves showing that, for these small tolerances, $y_{m}^{\#}$ is within the tolerance of the fixed point, $h_{m}\left(x_{0}\right)$. The desired principal result is then immediately obtained by combining this result with the result of Theorem 2.1, where it was shown that $\left\|h_{m}\left(x_{0}\right)-h\left(x_{0}\right)\right\|=\mathcal{O}\left(\varepsilon^{m+1}\right)$.

We begin by observing that

$$
\left\|y_{m}^{\#}-h_{m}\left(x_{0}\right)\right\| \leq\left\|y_{m}^{\#}-y^{(r)}\right\|+\left\|y^{(r)}-h_{m}\left(x_{0}\right)\right\|, \quad \text { for any } r>0,
$$

by the triangle inequality. The first term is $\mathcal{O}\left(\varepsilon^{m+1}\right)$ by definition, as long as $r$ is chosen large enough so that the stopping criterion, $\left\|y^{(r+1)}-y^{(r)}\right\|<\mathrm{TOL}_{m}$, is satisfied. As to the second term, we may obtain the same type of estimate, as follows: First,

$$
y^{(r+1)}-y^{(r)}=F_{m}\left(y^{(r)}\right)-y^{(r)}=-L_{m}\left(x_{0}, y^{(r)}\right),
$$

where we used equation (2.1), and hence

$$
L_{m}\left(x_{0}, y^{(r)}\right)=y^{(r)}-y^{(r+1)} .
$$

Second, $L_{m}$ is invertible in a neighborhood of its fixed point, by the Implicit Function Theorem, because the Jacobian of $L_{m}\left(x_{0}, \cdot\right)$ at $h_{m}\left(x_{0}\right)$ is

$$
\left(D_{y} L_{m}\right)\left(z_{m}\right)=\left(-\varepsilon^{-1} H D_{y} g\right)_{0}^{m+1},
$$

by equation (3.6), and $\operatorname{det}\left(D_{y} g\right)_{0} \neq 0$ since $\mathcal{L}_{[0]}$ is normally attracting. Third, by combining these first two observations, we see that

$$
y^{(r)}=L_{m}^{-1}\left(y^{(r)}-y^{(r+1)}\right),
$$

where $L_{m}^{-1}$ denotes the local inverse of $L_{m}\left(x_{0}, \cdot\right)$. Fourth, and finally, by expanding $L_{m}^{-1}$ around zero, noting that $L_{m}^{-1}(0)=h_{m}\left(x_{0}\right)$, and using the triangle inequality, we obtain

$$
\left\|y^{(r)}-h_{m}\left(x_{0}\right)\right\| \leq\left\|\left(D_{y} L_{m}^{-1}\right)(0)\right\|\left\|y^{(r)}-y^{(r+1)}\right\|+\mathcal{O}\left(\left\|y^{(r)}-y^{(r+1)}\right\|^{2}\right) .
$$

Recalling the stopping criterion, we have therefore obtained the desired bound on the second term, as well,

$$
\left\|y^{(r)}-h_{m}\left(x_{0}\right)\right\|<\left\|\left(D_{y} L_{m}^{-1}\right)(0)\right\| \mathrm{TOL}_{m}+\mathcal{O}\left(\left(\mathrm{TOL}_{m}\right)^{2}\right)
$$

Hence, the analysis of this section is complete.

\section{The EFFECTS OF DIFFERENCING}

In a numerical setting, the time derivatives of $y$ are approximated, at each iteration, by a differencing scheme,

$$
\left(\frac{\mathrm{d}^{m+1} y}{\mathrm{~d} t^{m+1}}\right)(z) \approx \frac{1}{\hat{H}^{m+1}}\left(\Delta^{m+1} y\right)(z), \quad \text { where } \quad z \equiv\left(x_{0}, y\right) \quad \text { and } \quad \hat{H}>0
$$


In this section, we examine how the approximation and convergence results of Sections $2-5$ are affected by the use of differencing. We choose forward differencing,

$$
\left(\Delta^{m+1} y\right)(z)=\sum_{k=0}^{m+1}(-1)^{m+1-k}\left(\begin{array}{c}
m+1 \\
k
\end{array}\right) \phi^{y}(z ; k \hat{H})
$$

where $\phi(z ; t)=\left(\left(\phi^{x}(z ; t)\right)^{\mathrm{T}},\left(\phi^{y}(z ; t)\right)^{\mathrm{T}}\right)^{\mathrm{T}}$ is the exact solution at time $t$ corresponding to the initial condition $z$ for concreteness of exposition, and where $\hat{H}$ is a positive, $\mathcal{O}(\varepsilon)$ quantity. Also, forward differencing is directly implementable in an equation-free or legacy code setting.

By the Mean Value Theorem,

$$
\begin{aligned}
\left(\Delta^{m+1} y\right)(z) & =\hat{H}^{m+1}\left(\frac{\mathrm{d}^{m+1} y}{\mathrm{~d} t^{m+1}}\right)(z)+\frac{m+1}{2} \hat{H}^{m+2}\left(\frac{\mathrm{d}^{m+2} y}{\mathrm{~d} t^{m+2}}\right)(\phi(z ; \hat{t})) \\
& =\left(-\frac{1}{\eta}\right)^{m+1}\left[L_{m}(z)-\frac{m+1}{2 \eta} L_{m+1}(\phi(z ; \hat{t}))\right]
\end{aligned}
$$

where $\eta=H / \hat{H}>0$ is an $\mathcal{O}(1)$ parameter available for tuning and $\phi(z ; \hat{t})$ is the point on the solution $\phi(z ; t)$ at some time $\hat{t} \in[0,(m+1) \hat{H}]$. Thus, for the $m$ th algorithm, the approximation of $\mathrm{d}^{m+1} y / \mathrm{d} t^{m+1}$ by the above scheme corresponds to generating the sequence $\left\{y^{(r)} \mid r=1,2, \ldots\right\}$ using the map

$$
\hat{F}_{m}(y)=y-\hat{L}_{m}(z), \quad z=\left(x_{0}, y\right)
$$

where

$$
\hat{L}_{m}(z)=(-\eta)^{m+1}\left(\Delta^{m+1} y\right)(z)=L_{m}(z)-\frac{m+1}{2 \eta} L_{m+1}(\phi(z ; \hat{t})) .
$$

Therefore, by equation (6.2),

$$
\hat{F}_{m}(y)=F_{m}(y)+\frac{m+1}{2 \eta} L_{m+1}(\phi(z ; \hat{t})) .
$$

Remark. For convenience in the analysis in this section, we take the flow $\phi$ to be the exact flow corresponding to equation (1.5). The analysis extends directly to many problems for which only a numerical approximation of $\phi$ is known. For example, if the discretization procedure admits a smooth error expansion (such as exists often for fixed step-size integrators in legacy codes or in the equation-free context), then the leading order results still hold, and the map $\phi$ obtained numerically is sufficiently accurate so that the remainder estimates below hold. In particular, given a $p$ th order scheme and an integration step size $\tilde{h}$, it suffices to take $\tilde{h}=\mathcal{O}(\varepsilon)$ to guarantee that the error made in using the numerically-obtained map $\phi$ is $\mathcal{O}\left(\varepsilon^{p}\right)$. Of course, with other integrators, one could alternatively require that the timestepper be $\mathcal{O}\left(\varepsilon^{m+2}\right)$ accurate, i.e., of one-higher order of accuracy.

\subsection{Existence of a fixed point $\hat{h}_{m}\left(x_{0}\right)$ of the map $\hat{F}_{m}$}

In this section, we establish that the map $\hat{F}_{m}$ has an isolated fixed point $y=\hat{h}_{m}\left(x_{0}\right)$ which differs only by terms of $\mathcal{O}\left(\varepsilon^{m+1}\right)$ from $h_{m}\left(x_{0}\right)$ (and thus also from $h\left(x_{0}\right)$, by virtue of Thm. 2.1).

The fixed point condition $\hat{F}_{m}\left(x_{0}, y\right)=y$ may be rewritten as

$$
0=\hat{L}_{m}\left(x_{0}, y\right)=L_{m}\left(x_{0}, y\right)-\frac{m+1}{2 \eta} L_{m+1}\left(\phi\left(x_{0}, y ; \hat{t}\right)\right)
$$

where we combined equations $(6.3)$ and (6.4). In order to show that $\hat{F}_{m}$ has an isolated fixed point $\hat{h}_{m}\left(x_{0}\right)$ which is $\mathcal{O}\left(\varepsilon^{m+1}\right)$ close to $h_{m}\left(x_{0}\right)$, we need to establish the validity of the following two conditions. 
(i). The second term in the right member of equation (6.5) satisfies the asymptotic estimate

$$
\left\|L_{m+1}\left(\phi\left(z_{m} ; \hat{t}\right)\right)\right\|=\mathcal{O}\left(\varepsilon^{m+1}\right), \quad \text { where } z_{m}=\left(x_{0}, h_{m}\left(x_{0}\right)\right) .
$$

(ii). The Jacobian of $\hat{L}_{m}$ satisfies

$$
\operatorname{det}\left(D_{y} \hat{L}_{m}\right)\left(z_{m}\right) \neq 0 \text { and } \quad\left\|\left(D_{y} \hat{L}_{m}\right)\left(z_{m}\right)\right\|_{2}=\mathcal{O}(1)
$$

Let us begin by examining the term $L_{m+1}\left(\phi\left(z_{m} ; \hat{t}\right)\right)$. Let $(\hat{x}, \hat{y})=\phi\left(z_{m} ; \hat{t}\right)$. Then, we may write

$$
L_{m+1}\left(\phi\left(z_{m} ; \hat{t}\right)\right)=L_{m+1}(\hat{x}, \hat{y})-L_{m+1}\left(\hat{x}, h_{m+1}(\hat{x})\right)
$$

because $L_{m+1}\left(\cdot, h_{m+1}(\cdot)\right) \equiv 0$ by the definition of $L_{m+1}$ and $h_{m+1}$. Hence,

$$
\left\|L_{m+1}\left(\phi\left(z_{m} ; \hat{t}\right)\right)\right\| \leq\left\|\left(D_{y} L_{m+1}\right)\left(\hat{x}, h_{m+1}(\hat{x})\right)\right\|\left\|\hat{y}-h_{m+1}(\hat{x})\right\|+\mathcal{O}\left(\left\|\hat{y}-h_{m+1}(\hat{x})\right\|^{2}\right) .
$$

Now, $\left\|\left(D_{y} L_{m+1}\right)\left(\hat{x}, h_{m+1}(\hat{x})\right)\right\|$ is $\mathcal{O}(1)$ by Lemma B.1. Next, the triangle inequality yields

$$
\left\|\hat{y}-h_{m+1}(\hat{x})\right\| \leq\|\hat{y}-h(\hat{x})\|+\left\|h(\hat{x})-h_{m+1}(\hat{x})\right\| .
$$

The first term in the right member remains $\mathcal{O}\left(\varepsilon^{m+1}\right)$ for all times $\left.\hat{t} \in[0,(m+1) \hat{H})\right]$. Indeed, the initial condition $z_{m}$ is $\mathcal{O}\left(\varepsilon^{m+1}\right)$ close to the normally attracting manifold $\mathcal{L}$. Thus, the Fenichel normal form [8] guarantees that the orbit generated by this initial condition remains $\mathcal{O}\left(\varepsilon^{m+1}\right)$ close to $\mathcal{L}$ for $\mathcal{O}(1)$ time intervals. The second term in the right member is also $\mathcal{O}\left(\varepsilon^{m+1}\right)$, by Theorem 2.1. Thus, $\left\|\hat{y}-h_{m+1}(\hat{x})\right\|$ is also $\mathcal{O}\left(\varepsilon^{m+1}\right)$. Substituting these estimations into inequality (6.8), we obtain that $\left\|L_{m+1}\left(\phi\left(z_{m} ; \hat{t}\right)\right)\right\|$ is $\mathcal{O}\left(\varepsilon^{m+1}\right)$ and condition (6.6) is satisfied.

Next, we determine the spectrum of $\left(D_{y} \hat{L}_{m}\right)\left(z_{m}\right)$ to leading order to check condition (6.7). We will work with the definition of $\Delta^{m+1} y$, equation (6.1), rather than with formula (6.2) which involves the unknown time $\hat{t}$. Combining equations (6.1) and (6.3), we obtain

$$
\hat{L}_{m}(z)=\eta^{m+1} \sum_{k=0}^{m+1}\left(\begin{array}{c}
m+1 \\
k
\end{array}\right)(-1)^{k} \phi^{y}(z ; k \hat{H})
$$

Differentiating both members of this equation with respect to $y$, we obtain

$$
\left(D_{y} \hat{L}_{m}\right)(z)=\eta^{m+1} \sum_{k=0}^{m+1}\left(\begin{array}{c}
m+1 \\
k
\end{array}\right)(-1)^{k}\left(D_{y} \phi^{y}\right)(z ; k \hat{H})
$$

Next, $\left(D_{y} \phi^{y}\right)\left(z_{m} ; t\right)=\mathrm{e}^{(t / \varepsilon)\left(D_{y} g\right)_{0}}$ to leading order for all $t$ of $\mathcal{O}(\varepsilon)$ because $\mathcal{L}$ is normally attracting. Since $k \hat{H}=\mathcal{O}(\varepsilon)$ for all $k=0,1, \ldots,(m+1)$, we may rewrite equation (6.9) to leading order as

$$
\left(D_{y} \hat{L}_{m}\right)\left(z_{m}\right)=\eta^{m+1} \sum_{k=0}^{m+1}\left(\begin{array}{c}
m+1 \\
k
\end{array}\right)\left(-\mathrm{e}^{(\hat{H} / \varepsilon)\left(D_{y} g\right)_{0}}\right)^{k}=\eta^{m+1}\left(I_{\mathrm{N}_{\mathrm{f}}}-\mathrm{e}^{(\hat{H} / \varepsilon)\left(D_{y} g\right)_{0}}\right)^{m+1} .
$$

Hence,

$$
\sigma\left(\left(D_{y} \hat{L}_{m}\right)\left(z_{m}\right)\right)=\left\{\eta^{m+1}\left(1-\mathrm{e}^{\lambda_{k} \hat{H} / \varepsilon}\right)^{m+1} \mid k=1, \ldots, \mathrm{N}_{\mathrm{f}}\right\},
$$

where $z_{m}=\left(x_{0}, h_{m}\left(x_{0}\right)\right)$. This leading order formula for the elements of the spectrum shows that $\left(D_{y} \hat{L}_{m}\right)\left(z_{m}\right)$ is $\mathcal{O}(1)$ and non-degenerate for all positive $\mathcal{O}(\varepsilon)$ values of $H$ and $\hat{H}$. Thus, condition (6.7) is also satisfied. 


\subsection{Stability of the fixed point $\hat{h}_{m}\left(x_{0}\right)$ for $\eta=1$}

In this section, we determine the stability of the fixed point $\hat{h}_{m}\left(x_{0}\right)$ under functional iteration using $\hat{F}_{m}$ in the case that $\hat{H}=H$. Our results for $\hat{H}=H$ are summarized in the following theorem. The general case $\hat{H} \neq H$ is treated in the next section, and the main result there is given in Theorem 6.2.

Theorem 6.1. Fix $\eta=1$. There is an $\varepsilon_{0}>0$ such that, for $0<\varepsilon \leq \varepsilon_{0}$, the functional iteration scheme defined by $\hat{F}_{0}$ is unconditionally stable. For each $m=1,2, \ldots$, there is an $\varepsilon_{m}>0$ such that, for $0<\varepsilon \leq \varepsilon_{m}$, the functional iteration scheme defined by $\hat{F}_{m}$ is stable if and only if, for each $k=1, \ldots, \mathrm{N}_{\mathrm{f}}$, the pair $\left(H, \theta_{k}\right)$ lies in the stability region the boundary of which is given by the implicit equation

$$
\begin{aligned}
1= & 2 \sum_{j=1}^{m+1} \sum_{\ell=1}^{j-1}\left(\begin{array}{c}
m+1 \\
j
\end{array}\right)\left(\begin{array}{c}
m+1 \\
\ell
\end{array}\right)(-1)^{j+\ell} \mathrm{e}^{-(j+\ell) H_{k}} \cos \left((j-\ell) H_{k} \tan \theta_{k}\right) \\
& +\sum_{\ell=1}^{m+1}\left(\begin{array}{c}
m+1 \\
\ell
\end{array}\right)^{2} \mathrm{e}^{-2 \ell H_{k}}, \text { where } H_{k}=-\lambda_{k, R} H / \varepsilon>0 .
\end{aligned}
$$

In particular, if $\lambda_{1}, \ldots, \lambda_{\mathrm{N}_{\mathrm{f}}}$ are real, then the functional iteration is unconditionally stable. If at least one of the eigenvalues has a nonzero imaginary part, then a sufficient and uniform (in $\theta_{1}, \ldots, \theta_{\mathrm{N}_{\mathrm{f}}}$ ) condition for stability is that

$$
H>\frac{\varepsilon H_{s}(1)}{\min _{k}\left|\lambda_{k, R}\right|}, \quad \text { where } H_{s}(1)=-\ln \left(2^{1 /(m+1)}-1\right) \geq 0 .
$$

The stability regions for various values of $m$ are plotted in Figure 2.

Following the procedure used in Section 3, we determine $\sigma\left(\left(D \hat{F}_{m}\right)\left(\hat{h}_{m}\left(x_{0}\right)\right)\right)$ and examine the circumstances in which the stability condition

is satisfied. Equation (6.3) yields

$$
\sigma\left(\left(D \hat{F}_{m}\right)\left(\hat{h}_{m}\left(x_{0}\right)\right)\right) \subset \mathrm{B}(0 ; 1)
$$

$$
\left(D \hat{F}_{m}\right)\left(\hat{h}_{m}\left(x_{0}\right)\right)=I_{\mathrm{N}_{\mathrm{f}}}-\left(D_{y} \hat{L}_{m}\right)\left(x_{0}, \hat{h}_{m}\left(x_{0}\right)\right)
$$

and thus also

$$
\left\{\hat{\mu}_{k}\right\} \equiv \sigma\left(\left(D_{y} \hat{F}_{m}\right)\left(\hat{h}_{m}\left(x_{0}\right)\right)\right)=1-\sigma\left(\left(D_{y} \hat{L}_{m}\right)\left(x_{0}, \hat{h}_{m}\left(x_{0}\right)\right)\right) .
$$

Since $\hat{h}_{m}\left(x_{0}\right)$ differs from $h_{m}\left(x_{0}\right)$ only at terms of $\mathcal{O}\left(\varepsilon^{m+1}\right), \quad\left(D_{y} \hat{L}_{m}\right)\left(x_{0}, \hat{h}_{m}\left(x_{0}\right)\right)$ also differs from $\left(D_{y} \hat{L}_{m}\right)\left(x_{0}, h_{m}\left(x_{0}\right)\right)$ only at terms of $\mathcal{O}\left(\varepsilon^{m+1}\right)$. Thus, equation (6.10) yields, to leading order and for $k=$ $1, \ldots, \mathrm{N}_{\mathrm{f}}$,

$$
\hat{\mu}_{k}=1-\left(1-\mathrm{e}^{\lambda_{k} H / \varepsilon}\right)^{m+1}=\sum_{\ell=1}^{m+1}\left(\begin{array}{c}
m+1 \\
\ell
\end{array}\right)(-1)^{\ell+1} \mathrm{e}^{\ell \lambda_{k} H / \varepsilon} .
$$

Recalling equation (3.1) and defining $H_{k}=-\lambda_{k, R} H / \varepsilon$, we rewrite equation (6.14) in the form

$$
\hat{\mu}_{k}=\sum_{\ell=1}^{m+1}\left(\begin{array}{c}
m+1 \\
\ell
\end{array}\right)(-1)^{\ell+1} \mathrm{e}^{-\ell H_{k}\left(1+i \tan \theta_{k}\right)}
$$

The stability condition (6.13) becomes, then,

$$
\left|\hat{\mu}_{k}\right|=\left|\sum_{\ell=1}^{m+1}\left(\begin{array}{c}
m+1 \\
\ell
\end{array}\right)(-1)^{\ell+1} \mathrm{e}^{-\ell H_{k}\left(1+i \tan \theta_{k}\right)}\right|<1, \quad \text { for all } k=1, \ldots, \mathrm{N}_{\mathrm{f}} .
$$



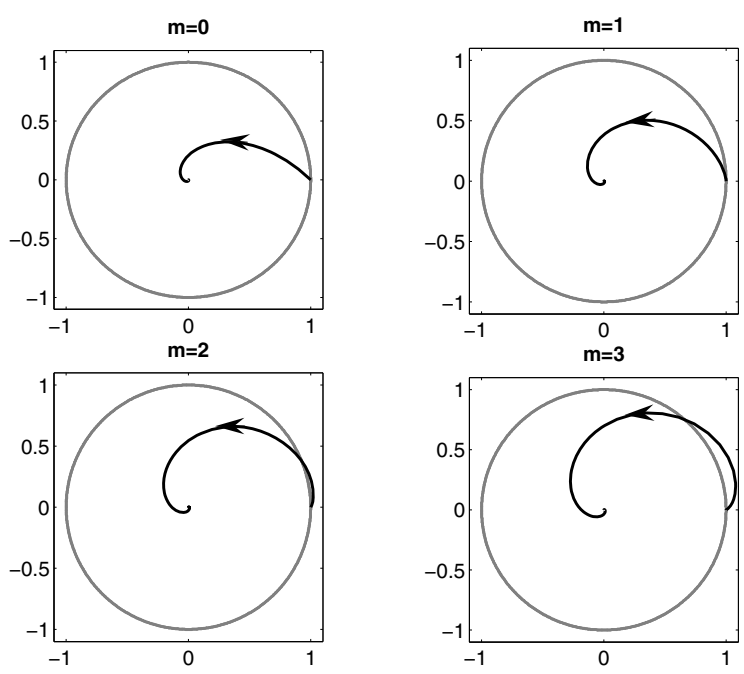

Figure 2. The eigenvalue $\hat{\mu}_{k}$ for values of $H$ between zero and $100 \varepsilon$. The thick line denotes the boundary of the stability region (i.e., the unit circle). The eigenvalue $\lambda_{k}$ was taken to be $-1+i$ for each one of the graphs. The arrow points to increasing values of $H$.

As in Section 3, we distinguish two cases.

Case 1. All of the eigenvalues of $\left(D_{y} g\right)_{0}$ are real. Then, $\theta_{k}=\pi$ for all $k=1, \ldots, \mathrm{N}_{\mathrm{f}}$, and hence equation (6.15) becomes

$$
\hat{\mu}_{k}=\sum_{\ell=1}^{m+1}\left(\begin{array}{c}
m+1 \\
\ell
\end{array}\right)(-1)^{\ell+1} \mathrm{e}^{-\ell H_{k}}=1-\left(1-\mathrm{e}^{-H_{k}}\right)^{m+1} .
$$

Thus, the spectrum of $\left(D_{y} \hat{F}_{m}\right)\left(\hat{h}_{m}\left(x_{0}\right)\right)$ is contained in $(0,1)$ for all positive $\mathcal{O}(\varepsilon)$ values of $H$. Equivalently, the fixed point $\hat{h}_{m}\left(x_{0}\right)$ is unconditionally stable for these values of $H$.

These results may be interpreted both in the context of the $m$ th iterative algorithm for each fixed $m$, as well as in the context of using the algorithms as an integrated class. In particular, for each fixed $m$, the rate of convergence to the fixed point of the $m$ th algorithm increases as $H$ increases. Also, for any fixed iterative step size $H$, the rate of convergence of the $m$ th algorithm to its fixed point decreases as the order, $m$, of the iterative algorithm increases. This information is important for determining how large an $H$ one should use, especially when using the algorithms as an integrated class.

Case 2. Some of the eigenvalues of $\left(D_{y} g\right)_{0}$ have nonzero imaginary parts. In this case, some of the eigenvalues may be unstable for certain values of $H$. Figure 2 demonstrates this: in it, we have drawn the complex eigenvalue $\hat{\mu}_{k}$ for various values of $H$ and for $m=0,1,2,3$. Plainly, $\hat{\mu}_{k}$ is unstable for $m>0$ and for $H$ small enough, as $\left|\hat{\mu_{k}}\right|>1$. We determine the stability regions in the $\left(\theta_{k}, H_{k}\right)$-plane as functions of $m$.

First, we derive the uniform bound (6.12). Using formula (6.15), we calculate

$$
\left|\hat{\mu}_{k}\right| \leq \sum_{\ell=1}^{m+1}\left(\begin{array}{c}
m+1 \\
\ell
\end{array}\right) \mathrm{e}^{-\ell H_{k}}=\left(1+\mathrm{e}^{-H_{k}}\right)^{m+1}-1
$$

and thus $\left|\hat{\mu}_{k}\right|<1$, for all $H_{k}>H_{s}(1)$. Recalling that $H_{k}=-\lambda_{k, R} H / \varepsilon$, we conclude that all of the eigenvalues $\hat{\mu}_{k}$ lie in the unit disk (equivalently, the $m$ th algorithm is stable) for all $\mathcal{O}(\varepsilon)$ values of $H$ greater than $\varepsilon H_{s}(1) / \min _{k}\left|\lambda_{k, R}\right|$, irrespective of the values of $\theta_{1}, \ldots, \theta_{\mathrm{N}_{\mathrm{f}}}$. This is demonstrated in Figure 3. 

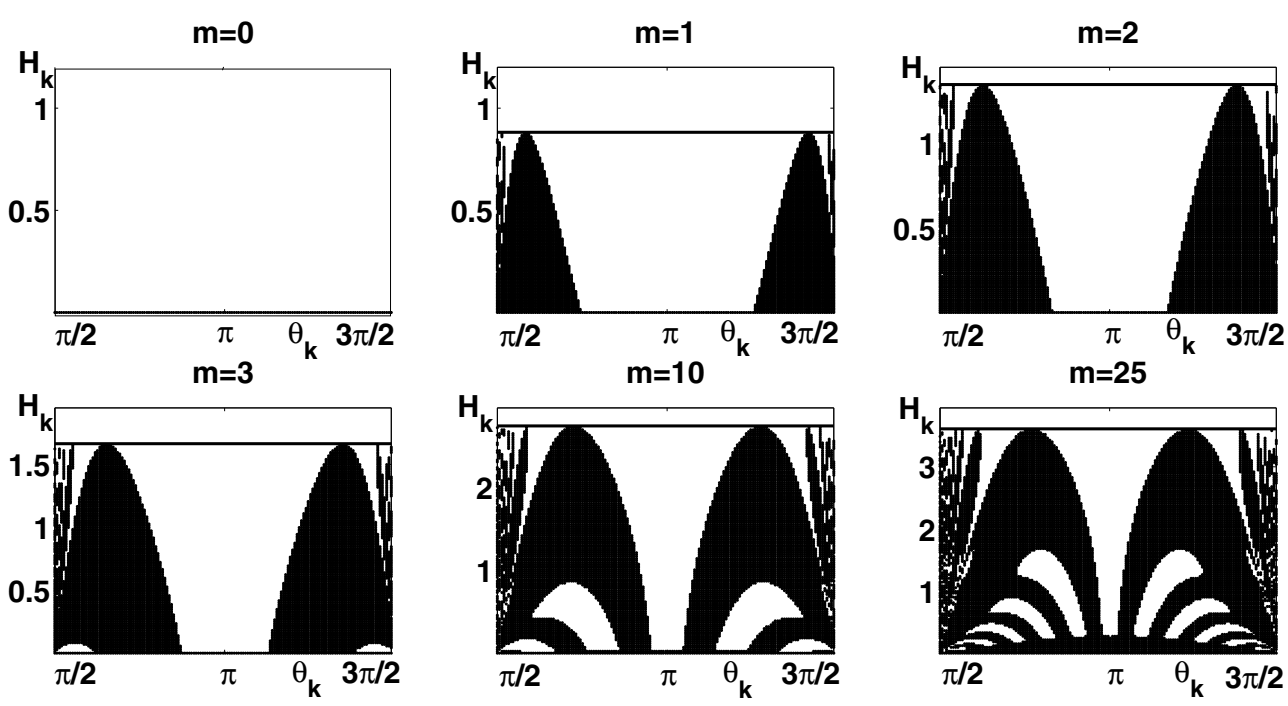

Figure 3. The regions of $H_{k}$ for which $\left|\mu_{k}\right|<1$ as functions of $\theta_{k} \in(\pi / 2,3 \pi / 2)$. White corresponds to stability $\left(\left|\mu_{k}\right|<1\right)$ and black to instability $\left(\left|\mu_{k}\right|>1\right)$. The angle $\theta_{k}$ takes values on $(\pi / 2,3 \pi / 2)$ and the black horizontal line corresponds to the uniform bound $H_{s}(1)$ of equation (6.12).

Next, we derive formulae which describe exactly the stability regions. For $m=0$, equation (6.12) yields $H_{s}(1)=0$. Thus, $\left|\hat{\mu}_{k}\right|<1$ for all positive $\mathcal{O}(\varepsilon)$ values of $H$ and for all $k=1, \ldots, \mathrm{N}_{\mathrm{f}}$. As a result, the fixed point $\hat{h}_{0}\left(x_{0}\right)$ is unconditionally stable for positive, $\mathcal{O}(\varepsilon)$ values of $H$, see also Figure 3 .

For $m=1$, equation (6.15) becomes

$$
\hat{\mu}_{k}=2 \mathrm{e}^{-H_{k}\left(1+\mathrm{i} \tan \theta_{k}\right)}-\mathrm{e}^{-2 H_{k}\left(1+\mathrm{i} \tan \theta_{k}\right)} .
$$

Writing $\overline{\hat{\mu}_{k}}$ for the complex conjugate of $\hat{\mu}_{k}$, then, we calculate

$$
\left|\hat{\mu}_{k}\right|^{2}=\hat{\mu}_{k} \overline{\hat{\mu}_{k}}=4 \mathrm{e}^{-2 H_{k}}-4 \mathrm{e}^{-3 H_{k}} \cos \left(H_{k} \tan \theta_{k}\right)+\mathrm{e}^{-4 H_{k}} .
$$

Using this formula, we recast the stability condition (6.16) into the form

$$
4 \mathrm{e}^{-2 H_{k}}-4 \mathrm{e}^{-3 H_{k}} \cos \left(H_{k} \tan \theta_{k}\right)+\mathrm{e}^{-4 H_{k}}<1
$$

In particular, the boundary of the stability region can be obtained by equating the expression in the left member of this inequality to one and solving for $\theta_{k}$, to obtain

$$
\theta_{k}=\arctan \left(H_{k}^{-1}\left[\arccos \left[\frac{1}{4} \mathrm{e}^{-H_{k}}+\mathrm{e}^{H_{k}}-\frac{1}{4} \mathrm{e}^{3 H_{k}}\right]+2 \ell \pi\right]\right) .
$$

Here, $\ell \in \mathbf{Z}$ and the branch of arctan is chosen so that $\theta_{k} \in(\pi / 2,3 \pi / 2)$. We have plotted the stability region in Figure 3. We also note here that the boundary of the stability region close to $\pi / 2$ and to $3 \pi / 2$ has fine structure, see Figure 4.

For a general value of $m$, the stability condition (6.16) is

$$
\left|\hat{\mu}_{k}\right|=\left|\sum_{\ell=1}^{m+1}\left(\begin{array}{c}
m+1 \\
\ell
\end{array}\right)(-1)^{\ell+1} \mathrm{e}^{-\ell H_{k}\left(1+i \tan \theta_{k}\right)}\right|<1, \quad \text { for all } k=1, \ldots, \mathrm{N}_{\mathrm{f}} .
$$




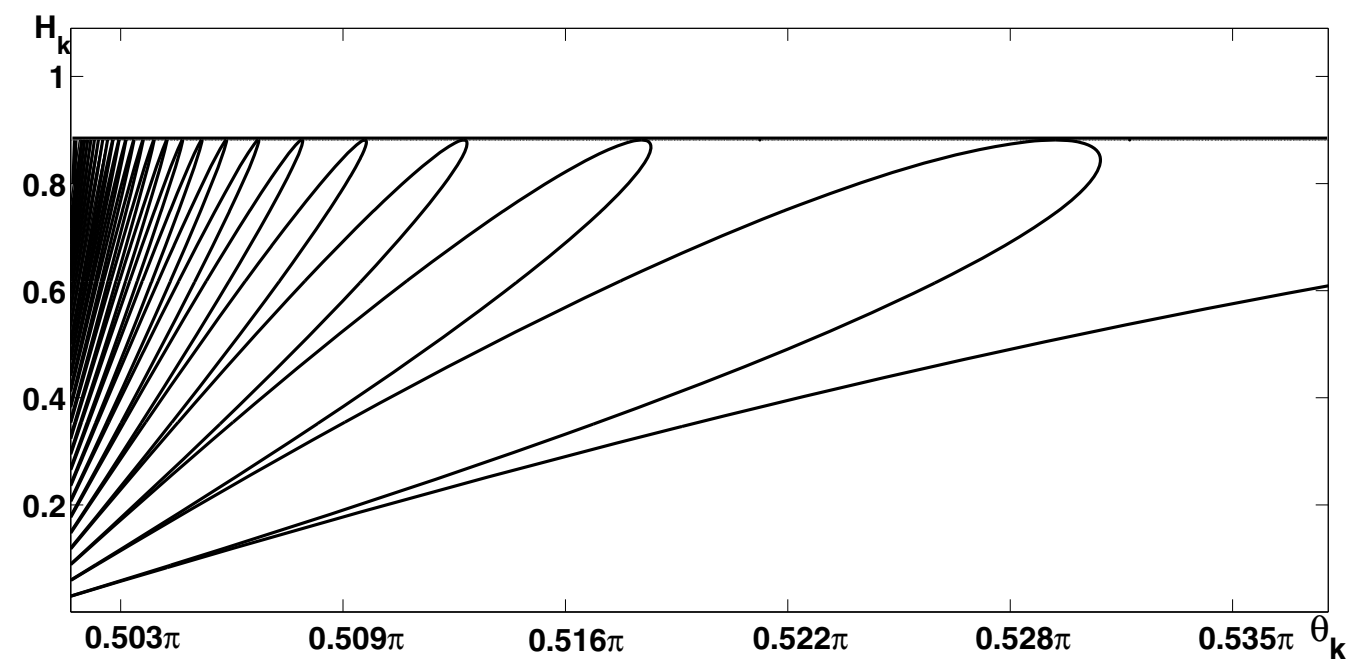

FiguRE 4. The fine structure of the stability region depicted in Figure 3 (with $m=1$ ) close to $\pi / 2$. The exterior of the lobes is part of the stability region.

Now, using equation (6.15), we calculate

$$
\begin{aligned}
\left|\hat{\mu}_{k}\right|^{2}= & \hat{\mu}_{k} \overline{\hat{\mu}}_{k} \\
= & \sum_{j=1}^{m+1} \sum_{\ell=1}^{m+1}\left(\begin{array}{c}
m+1 \\
j
\end{array}\right)\left(\begin{array}{c}
m+1 \\
\ell
\end{array}\right)(-1)^{j+\ell} \mathrm{e}^{-(j+\ell) H_{k}} \mathrm{e}^{i(j-\ell) H_{k} \tan \theta_{k}} \\
= & 2 \sum_{j=1}^{m+1} \sum_{\ell=1}^{j-1}\left(\begin{array}{c}
m+1 \\
j
\end{array}\right)\left(\begin{array}{c}
m+1 \\
\ell
\end{array}\right)(-1)^{j+\ell} \mathrm{e}^{-(j+\ell) H_{k}} \cos \left((j-\ell) H_{k} \tan \theta_{k}\right) \\
& +\sum_{\ell=1}^{m+1}\left(\begin{array}{c}
m+1 \\
\ell
\end{array}\right)^{2} \mathrm{e}^{-2 \ell H_{k}} .
\end{aligned}
$$

Equation (6.11) now follows directly.

\subsection{Stability of the fixed point $\hat{h}_{m}\left(x_{0}\right)$ for $\eta \neq 1$}

In this section, we determine the stability of the fixed point $\hat{h}_{m}\left(x_{0}\right)$ for $\hat{H} \neq H$. We define the function

$$
\hat{H}_{m}(\eta)=\left\{\begin{array}{llr}
-\ln \left(2^{1 /(m+1)}-1\right), & \text { if } & 0<\eta \leq 1 \\
-\ln \left|2^{1 /(m+1)} / \eta-1\right|, & \text { if } & \eta>1
\end{array}\right.
$$

Our results are summarized in the following theorem.

Theorem 6.2. Fix $\eta>0$. For each $m=0,1,2, \ldots$, there is an $\varepsilon_{m}>0$ such that, for $0<\varepsilon \leq \varepsilon_{m}$, the functional iteration scheme defined by $\hat{F}_{m}$ is stable if and only if, for each $k=1, \ldots, \mathrm{N}_{\mathrm{f}}$, the pair $\left(\hat{H}, \theta_{k}\right)$ lies 
in the stability region the boundary of which is given by the implicit equation

$$
\begin{aligned}
1= & 2 \eta^{2(m+1)} \sum_{j=1}^{m+1} \sum_{\ell=1}^{j-1}\left(\begin{array}{c}
m+1 \\
j
\end{array}\right)\left(\begin{array}{c}
m+1 \\
\ell
\end{array}\right)(-1)^{j+\ell} \mathrm{e}^{-(j+\ell) \hat{H}_{k}} \cos \left((j-\ell) \hat{H}_{k} \tan \theta_{k}\right) \\
& +2 \eta^{m+1}\left(\eta^{m+1}-1\right) \sum_{\ell=1}^{m+1}\left(\begin{array}{c}
m+1 \\
\ell
\end{array}\right)(-1)^{\ell} \mathrm{e}^{-\ell \hat{H}_{k}} \cos \left(\ell \hat{H}_{k} \tan \theta_{k}\right) \\
& +\eta^{2(m+1)} \sum_{\ell=1}^{m+1}\left(\begin{array}{c}
m+1 \\
\ell
\end{array}\right)^{2} \mathrm{e}^{-2 \ell \hat{H}_{k}}+\left(\eta^{m+1}-1\right)^{2},
\end{aligned}
$$

where $\hat{H}_{k}=-\lambda_{k, R} \hat{H} / \varepsilon>0$. In particular:

(i) Assume that $\operatorname{Im}\left(\lambda_{k}\right)=0$, for all $k=1, \ldots, \mathrm{N}_{\mathrm{f}}$. If $0<\eta<2^{1 /(m+1)}$, then the functional iteration is unconditionally stable. If $\eta>2^{1 /(m+1)}$, then the functional iteration is stable if and only if

$$
0<\hat{H}<\frac{\varepsilon \hat{H}_{m}(\eta)}{\max _{k}\left|\lambda_{k, R}\right|}
$$

(ii) Assume that at least one of $\operatorname{Im}\left(\lambda_{1}\right), \ldots, \operatorname{Im}\left(\lambda_{\mathrm{N}_{\mathrm{f}}}\right)$ is nonzero. If $0<\eta<2^{1 /(m+1)}$, then a sufficient and uniform (in $\theta_{1}, \ldots, \theta_{\mathrm{N}_{\mathrm{f}}}$ ) condition for stability is

$$
\hat{H}>\frac{\varepsilon \hat{H}_{m}(\eta)}{\min _{k}\left|\lambda_{k, R}\right|} .
$$

If $\eta>2^{1 /(m+1)}$, the functional iteration is unstable for any $\theta_{1}, \ldots, \theta_{\mathrm{N}_{\mathrm{f}}}$ and for all

$$
\hat{H}>\frac{\varepsilon \hat{H}_{m}(\eta)}{\max _{k}\left|\lambda_{k, R}\right|} \text {. }
$$

These results are demonstrated in Figures 5, 6 and 7 .

As in Section 6.2, we determine when the stability condition (6.13) holds. The analogue of equations (6.14) and (6.15) in this case is, to leading order and for $k=1, \ldots, \mathrm{N}_{\mathrm{f}}$,

$$
\hat{\mu}_{k}=1-\eta^{m+1}\left(1-\mathrm{e}^{\lambda_{k} \hat{H} / \varepsilon}\right)^{m+1}=1-\eta^{m+1}\left(1-\mathrm{e}^{-\hat{H}_{k}\left(1+i \tan \theta_{k}\right)}\right)^{m+1} .
$$

The stability condition (6.13) becomes, then,

$$
\left|\hat{\mu}_{k}\right|=\left|1-\eta^{m+1}\left(1-\mathrm{e}^{-\hat{H}_{k}\left(1+i \tan \theta_{k}\right)}\right)^{m+1}\right|<1, \quad \text { for all } k=1, \ldots, \mathrm{N}_{\mathrm{f}} .
$$

Here also, we distinguish two cases.

Case 1. All of the eigenvalues of $\left(D_{y} g\right)_{0}$ are real. Then, $\theta_{k}=\pi$ for all $k=1, \ldots, \mathrm{N}_{\mathrm{f}}$, and hence equation (6.25) becomes

$$
\hat{\mu}_{k}=1-\eta^{m+1}\left(1-\mathrm{e}^{-\hat{H}_{k}}\right)^{m+1} .
$$

Plainly, the condition $\hat{\mu}_{k}<1$ is satisfied for all positive $\hat{H}_{k}$ and $\eta$. Next, solving this equation for $\eta$, we obtain an equation for the level curve $\hat{\mu}_{k}=$ const.,

$$
\eta=\frac{\left(1-\hat{\mu}_{k}\right)^{1 /(m+1)}}{1-\mathrm{e}^{-\hat{H}_{k}}}
$$




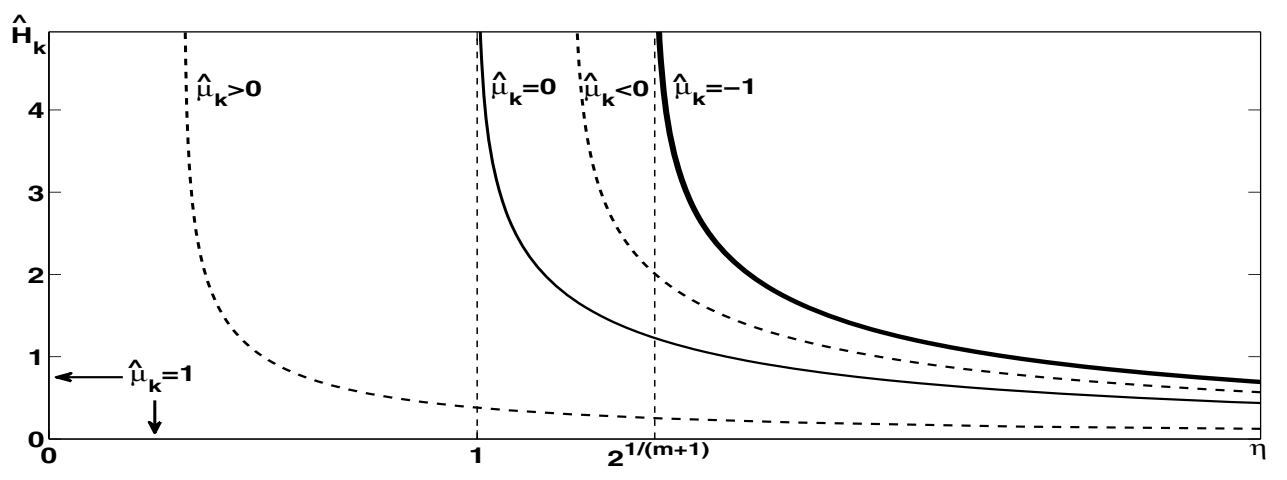

Figure 5. The stability region in the $\left(\eta, \hat{H}_{k}\right)$-plane together with level curves of the eigenvalue $\hat{\mu}_{k}\left(\eta, \hat{H}_{k}\right)$ for the case of a real $\lambda_{k}$. The eigenvalue $\hat{\mu}_{k}$ is stable for all pairs $\left(\eta, \hat{H}_{k}\right)$ to the left of the level curve $\hat{\mu}_{k}=-1$ (thick curve).

For $0<\eta<2^{1 /(m+1)}$ and for all $\mathcal{O}(\varepsilon)$ and positive values of $\hat{H}$, we obtain $\hat{\mu}_{k}>-1$ (and thus the eigenvalue $\hat{\mu}_{k}$ is stable), see Figure 5. Therefore, $\sigma\left(\left(D_{y} \hat{F}_{m}\right)\left(\hat{h}_{m}\left(x_{0}\right)\right)\right) \subset(-1,1)$, and the fixed point $\hat{h}_{m}\left(x_{0}\right)$ is unconditionally stable.

For $\eta>2^{1 /(m+1)}$, we obtain the condition $0<\hat{H}_{k}<\hat{H}_{m}(\eta)$, and equation (6.21) follows directly. Finally, we note that, for a fixed value of $\eta$ and as $\hat{H} \rightarrow \infty$, the spectrum clusters around $1-\eta^{m+1}$. Thus, the choice $\eta=1$ is optimal in the sense that large values of $\hat{H}$ bring the spectrum closer to zero.

Case 2. Some of the eigenvalues of $\left(D_{y} g\right)_{0}$ have nonzero imaginary parts. In this case, some of the eigenvalues may become unstable for certain combinations of $\eta$ and $\hat{H}$, as our analysis in Section 6.2 also showed.

First, we consider the case $0<\eta<2^{1 /(m+1)}$ and derive the uniform bound (6.22). Using formula (6.24) and working as in equation (6.17), we estimate

$$
\left|\hat{\mu}_{k}\right| \leq\left|1-\eta^{m+1}\right|+\eta^{m+1}\left[\left(1+\mathrm{e}^{-\hat{H}_{k}}\right)^{m+1}-1\right] .
$$

Hence,

$$
\left|\hat{\mu}_{k}\right| \leq \begin{cases}1+\eta^{m+1}\left[\left(1+\mathrm{e}^{-\hat{H}_{k}}\right)^{m+1}-2\right], & \text { for } \quad 0<\eta \leq 1, \\ \eta^{m+1}\left(1+\mathrm{e}^{-\hat{H}_{k}}\right)^{m+1}-1, & \text { for } \quad \eta>1 .\end{cases}
$$

Combining these inequalities with the stability condition $\left|\hat{\mu}_{k}\right|<1$, we obtain the sufficient condition $\hat{H}_{k}>$ $\hat{H}_{m}(\eta)$, where $\hat{H}_{m}(\eta)$ is the uniform bound (6.19) (see also Fig. 6). Recalling that $\hat{H}_{k}=-\lambda_{k, R} \hat{H} / \varepsilon$, we conclude that, if condition (6.22) is satisfied, then $\sigma\left(\left(D_{y} \hat{F}_{m}\right)\left(\hat{h}_{m}\left(x_{0}\right)\right)\right) \subset \mathrm{B}(0 ; 1)$, and hence the $m$ th algorithm is stable.

Next, we consider the case $\eta>2^{1 /(m+1)}$ and derive the uniform bound (6.23). Equation (6.24) yields

$$
\left|1-\hat{\mu}_{k}\right| \geq \eta^{m+1}\left(1-\left|\mathrm{e}^{-\hat{H}_{k}} \mathrm{e}^{i \hat{H}_{k} \tan \theta_{k}}\right|\right)^{m+1} \geq \eta^{m+1}\left(1-\mathrm{e}^{-\hat{H}_{k}}\right)^{m+1} .
$$

Thus, $\left|1-\hat{\mu}_{k}\right|>2$, for $\eta>2^{1 /(m+1)}$ and $\hat{H}_{k}>\hat{H}_{m}(\eta)$, and therefore

$$
\left|\hat{\mu}_{k}\right| \geq|| 1-\hat{\mu}_{k}|-1|>1
$$

Hence, $\hat{\mu}_{k}$ is unstable. 

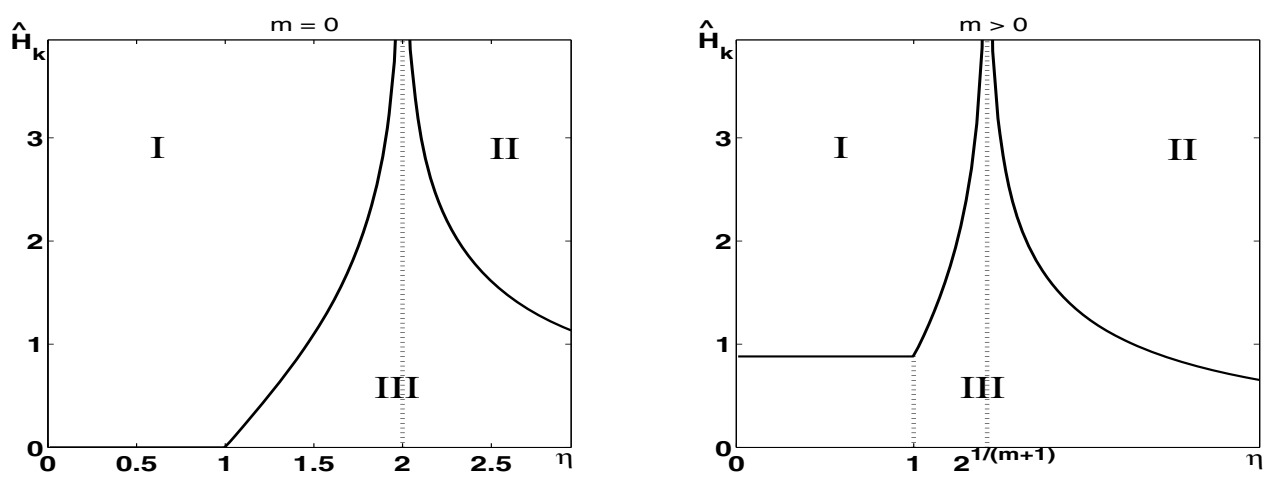

Figure 6 . The stability regions in the $\left(\eta, \hat{H}_{k}\right)$-plane for $m=0$ (left panel) and $m=1,2, \ldots$ (right panel). The eigenvalue $\hat{\mu}_{k}$ is stable in region I, unstable in region II, and its stability type is $\theta_{k}$-dependent in region III.

Remark. Conditions (6.22) and (6.23) may be interpreted by means of the fact that $\sigma\left(\left(D_{y} \hat{F}_{m}\right)\left(\hat{h}_{m}\left(x_{0}\right)\right)\right)$ clusters around $1-\eta^{m+1}$ as $\hat{H} \rightarrow \infty$. For $0<\eta<2^{1 /(m+1)}$, there holds that $-1<1-\eta^{m+1}<1$. Thus, for $\hat{H}$ large enough, the eigenvalues are contained in the unit disk. On the contrary, $1-\eta^{m+1}<-1$ for $\eta>2^{1 /(m+1)}$, and thus the eigenvalues lie outside the unit disk for $\hat{H}$ large enough.

Finally, formula (6.20) describing the stability region may be derived in a manner entirely analogous to that used to derive equation (6.11).

\section{COnClusions And Discussion}

In this article, we characterized the accuracy and convergence properties of the class of iterative algorithms introduced in [6] for explicit fast-slow systems (1.5). The $m$ th member of the class corresponds to a functional iteration scheme to solve the $(m+1)$ st derivative condition (1.6). We showed that this condition has an isolated solution, which corresponds to a fixed point of this $m$ th member and which is accurate up to and including terms of $\mathcal{O}\left(\varepsilon^{m}\right)$, see Theorem 2.1. Also, we derived explicit formulae for the domain of convergence of the functional iteration, both in the case where analytical formulae for the $(m+1)$ st derivative are used (see Thm. 3.1) and in the case where the $(m+1)$ st derivatives are estimated through a forward difference scheme (see Thms. 6.1 and 6.2). These convergence results are illustrated in Figures 1, 3, 4, 5 and 6. Further, we demonstrated how the Recursive Projection Method may be used to stabilize the functional iteration in all cases when it is unstable or to accelerate its convergence in those cases where the convergence is slow.

An extension of the analysis presented here to more general multiscale systems (1.1) is presented in [22]. The analysis of the accuracy of the $(m+1)$ st derivative condition presented in Section 2 carries through, essentially (modulo a number of technicalities), in the more general case as well. The analysis of the stability of the functional iteration, on the other hand, is far more involved. The reason for that is that, although the hyperplane $u=u_{0}$ and the space tangent to the fast fibration over the slow manifold coincide to leading order for explicit fast-slow systems (1.5), this is not the case for the more general systems (1.1). The absence of this feature makes the stability question for the functional iteration far more difficult to answer in the general case.

In addition, we are in the process of generalizing the results of this article to other maps that may be used in the context of the functional iteration scheme developed in [6]. In particular, it is of interest to use maps which are implicitly defined (as opposed to the explicitly defined ones presented in [6] and in this article). Preliminary analytical results for $m=0$ and $m=1$ indicate that one may construct functional iteration schemes based on implicit maps which not only retain the accuracy of the functional iteration scheme presented in this article but which are also unconditionally stable. Moreover, we think that this analysis may be extended to higher values 

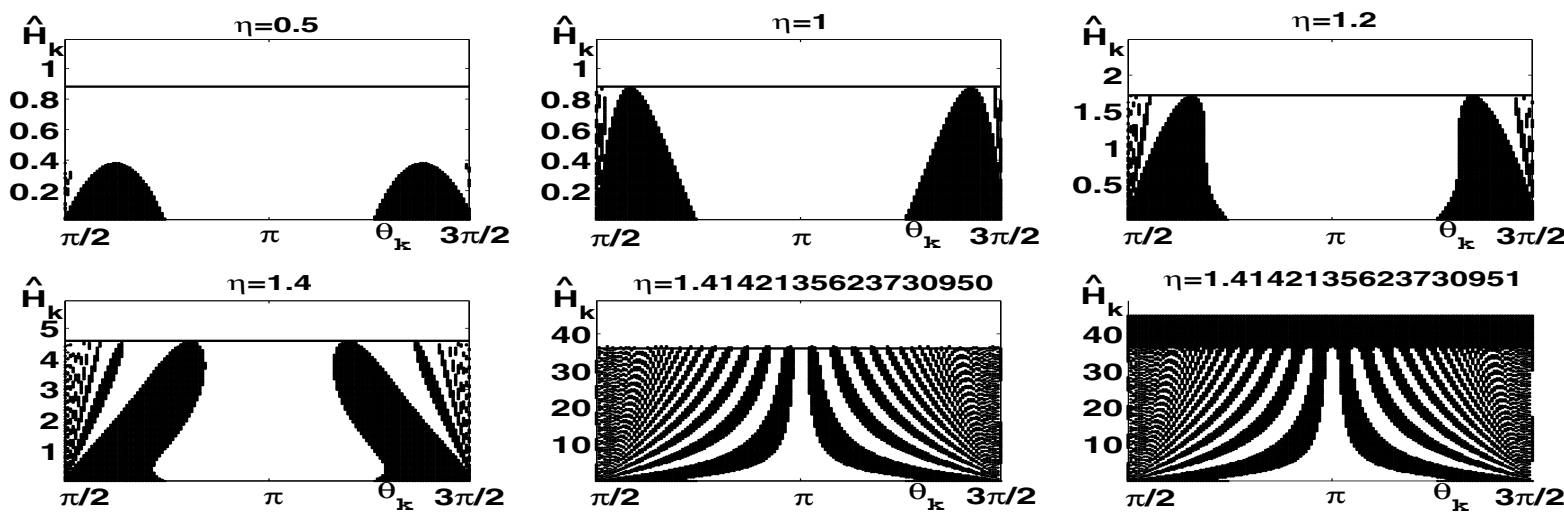

Figure 7 . The stability region in the $\left(\theta_{k}, \hat{H}_{k}\right)$-plane for $m=1$ and for various values of $\eta$. The last two values for $\eta$ are just below and just above the value $2^{1 /(m+1)}=\sqrt{2}$. White denotes stability and black denotes instability.

of $m$, and we note that it is also possible to carry out the functional iteration with implicitly defined maps even when one only has a legacy code as a timestepper.

\section{Appendix A. The OnE-HIGHER-ORDER PROPOSITION}

In this appendix, we state and prove a technical proposition - called the one-higher-order proposition - about the asymptotic accuracy of approximations of $\mathcal{L}$ given an approximation of the normal space to $\mathcal{L}$. This result is instrumental in the proof of the technical lemmata contained in the next appendix.

We begin by recalling the useful formulation, equation (1.11), of the invariance equation that defines the function $h$, whose graph is the invariant, slow manifold $\mathcal{L}$. This formulation revealed that the matrix $\left(-D h(x), I_{N_{f}}\right)$ forms a basis for $\mathrm{N}_{z} \mathcal{L}$, the space normal to the slow manifold at the point $z=(x, h(x)) \in \mathcal{L}$. The one-higherorder proposition, which we now state and prove, establishes a connection between the order in $\varepsilon$ to which a set $N$ of row vectors approximates $\mathrm{N}_{z} \mathcal{L}$ and the order to which the solution $\eta$ to the condition $N G=0$ approximates $h$.

Proposition A.1. Let $\varepsilon>0$ be sufficiently small. Let $N(x, \varepsilon)$ be an $\mathrm{N}_{\mathrm{f}} \times \mathrm{N}$ matrix with the property that its rows span $\mathrm{N}_{z} \mathcal{L}$ up to and including terms of $\mathcal{O}\left(\varepsilon^{m}\right)$, for some $m=0,1, \ldots$ That is, $N(\cdot, \varepsilon)$ is of the form

$$
N(\cdot, \varepsilon)=C\left(-\sum_{i=0}^{m} \varepsilon^{i} D h_{[i]}(\cdot)-\sum_{i \geq m+1} \varepsilon^{i} R_{i}(\cdot), I_{\mathrm{N}_{\mathrm{f}}}\right),
$$

where $C$ is a non-singular $\mathrm{N}_{\mathrm{f}} \times \mathrm{N}_{\mathrm{f}}$ matrix and $R_{i} \neq D h_{[i]}$, for $i=m+1, m+2, \ldots$, in general. Then, the condition

$$
N(x, \varepsilon) G(x, y, \varepsilon)=0
$$

can be solved for $y$ to yield a function $y=\eta(x)$, the asymptotic expansion of which agrees with that of $h(x)$ up to and including terms of $\mathcal{O}\left(\varepsilon^{m+1}\right)$,

$$
\eta(x)=\sum_{i=0} \varepsilon^{i} \eta_{i}(x)=\sum_{i=0}^{m+1} \varepsilon^{i} h_{[i]}(x)+\mathcal{O}\left(\varepsilon^{m+2}\right)
$$


This proposition is called the one-higher-order proposition, because it states that the order to which $\eta(x)$ approximates the full slow manifold is of one higher than that to which $N$ approximates the normal space.

Proof of Proposition A.1. We recall that $h(\cdot)=\Sigma_{i=0} \varepsilon^{i} h_{[i]}(\cdot)$, by equation (1.12), and that $h_{[i]}$ is determined from the $\mathcal{O}\left(\varepsilon^{i}\right)$ terms of the invariance equation (1.11). Similarly, $\eta_{i}$ is determined from the $\mathcal{O}\left(\varepsilon^{i}\right)$ terms of equation (A.2). Thus, to establish equation (A.3), it suffices to compare the terms of these two equations from $\mathcal{O}(1)$ up through and including $\mathcal{O}\left(\varepsilon^{m+1}\right)$ and to show that they are equal.

First, for each $i=0,1, \ldots, m$, the invariance equation (1.11) at $\mathcal{O}\left(\varepsilon^{i}\right)$ is

$$
\left(-D h_{[0]}, I_{\mathrm{N}_{\mathrm{f}}}\right) G_{i}+\sum_{\ell=1}^{i}\left(-D h_{[\ell]}, 0\right) G_{i-\ell}=0 .
$$

Second, to derive the $\mathcal{O}\left(\varepsilon^{i}\right)$ terms for the condition $N G=0$, we substitute the hypothesis (A.1) in equation (A.2) and left-multiply by $C^{-1}$ to obtain

$$
C^{-1} N G=\left(-\sum_{i=0}^{m} \varepsilon^{i} D h_{[i]}+\mathcal{O}\left(\varepsilon^{m+1}\right), I_{\mathrm{N}_{\mathrm{f}}}\right) G=0 .
$$

Thus, for each $i=0,1, \ldots, m$, this condition at $\mathcal{O}\left(\varepsilon^{i}\right)$ is

$$
\left(-D h_{[0]}, I_{\mathrm{N}_{\mathrm{f}}}\right) G_{i}+\sum_{\ell=1}^{i}\left(-D h_{[\ell]}, 0\right) G_{i-\ell}=0 .
$$

Plainly, this equation is identical to equation (A.4). Thus, proceeding inductively in $i$, one has $\eta_{i}=h_{[i]}$, for $i=0,1, \ldots, m$.

Finally, we look at the $\mathcal{O}\left(\varepsilon^{m+1}\right)$ terms of the two equations. Equation (A.4) with $i=m+1$ is

$$
\left(-D h_{[0]}, I_{\mathrm{N}_{\mathrm{f}}}\right) G_{m+1}+\sum_{\ell=1}^{m}\left(-D h_{[\ell]}, 0\right) G_{m+1-\ell}+\left(-D h_{[m+1]}, 0\right) G_{0}=0 .
$$

Also, equation (A.5) at $\mathcal{O}\left(\varepsilon^{m+1}\right)$ is

$$
\left(-D h_{[0]}, I_{\mathrm{N}_{\mathrm{f}}}\right) G_{m+1}+\sum_{\ell=1}^{m}\left(-D h_{[\ell]}, 0\right) G_{m+1-\ell}+\left(R_{m+1}, 0\right) G_{0}=0 .
$$

We note that $R_{m+1} \neq-D h_{[m+1]}$, in general. However, $G_{0}=0$ in equations (A.6)-(A.7), since this term is evaluated at $\left(x, \eta_{0}, 0\right)=\left(x, h_{[0]}, 0\right)$. Thus, equations (A.6) and (A.7) also agree, and hence $\eta_{m+1}=h_{[m+1]}$. This completes the proof of the proposition.

\section{Appendix B. Proofs of Lemmata 2.1 And 2.2}

In this appendix, we prove Lemmata 2.1 and 2.2 characterizing the asymptotic accuracy of the approximation to $\mathcal{L}$ obtained from the $(m+1)$ st derivative condition $(2.10)$.

Proof of Lemma 2.1. We write $z_{m}$ for $\left(x, h_{m}(x)\right)$ and $z$ for $(x, h(x))$. The strategy is as follows: We will show that the rows of $\left(D_{z} L_{m}\right)\left(z_{m}, \varepsilon\right)$ span $\mathrm{N}_{z} \mathcal{L}$ up to and including terms of $\mathcal{O}\left(\varepsilon^{m}\right)$. Then, we will apply Proposition A.1 to establish equation (2.12).

The manifold $\mathcal{L}_{m}$ is the graph of the function $h_{m}$, and thus it coincides exactly with the zero level set of the function $-h_{m}(\cdot)+y$. As a result, the rows of the $\mathrm{N}_{\mathrm{f}} \times \mathrm{N}$ gradient matrix $\left(-D h_{m}(x), I_{\mathrm{N}_{\mathrm{f}}}\right)$ form a basis 
for $\mathrm{N}_{z_{m}} \mathcal{L}_{m}$. Second, the function $h_{m}(\cdot)$ is defined through the $(m+1)$ st derivative condition $L_{m}\left(\cdot, h_{m}(\cdot), \varepsilon\right)=0$. Therefore, $\mathcal{L}_{m}$ also coincides with (a connected component of) the zero level set of the function $L_{m}(\cdot, \varepsilon)$. Thus, the rows of the $\mathrm{N}_{\mathrm{f}} \times \mathrm{N}$ gradient matrix $\left(D_{z} L_{m}\right)\left(x, h_{m}(x), \varepsilon\right)$ also form a basis for $\mathrm{N}_{z_{m}} \mathcal{L}_{m}$. It follows that there exists a non-singular $\mathrm{N}_{\mathrm{f}} \times \mathrm{N}_{\mathrm{f}}$ matrix $C$ such that

$$
\left(D_{z} L_{m}\right)\left(\cdot, h_{m}(\cdot), \varepsilon\right)=C\left(-D h_{m}(\cdot), I_{\mathrm{N}_{\mathrm{f}}}\right) .
$$

Next, the induction hypothesis implies that the asymptotic expansions of $h_{m}$ and $h$ agree up to and including terms of $\mathcal{O}\left(\varepsilon^{m}\right)$,

$$
h_{m}(\cdot)=\sum_{i=0}^{m} \varepsilon^{i} h_{[i]}(\cdot)+\mathcal{O}\left(\varepsilon^{m+1}\right) .
$$

Since the vector field is assumed to be sufficiently smooth, we may differentiate both sides of this equation with respect to the argument to obtain

$$
D h_{m}(\cdot)=\sum_{i=0}^{m} \varepsilon^{i} D h_{[i]}(\cdot)+\mathcal{O}\left(\varepsilon^{m+1}\right),
$$

where the remainder stays of the same order due to smoothness. Combining equations (B.1) and (B.3), then, we find

$$
\left(D_{z} L_{m}\right)\left(\cdot, h_{m}(\cdot), \varepsilon\right)=C\left(-\sum_{i=0}^{m} \varepsilon^{i} D h_{[i]}(\cdot)+\mathcal{O}\left(\varepsilon^{m+1}\right), I_{\mathrm{N}_{\mathrm{f}}}\right) .
$$

This equation shows that the rows of $\left(D_{z} L_{m}\right)\left(x, h_{m}(x), \varepsilon\right) \operatorname{span} \mathrm{N}_{z} \mathcal{L}$ up to and including terms of $\mathcal{O}\left(\varepsilon^{m}\right)$. Hence, application of the one-higher-order proposition, Proposition A.1, completes the proof of this lemma.

Before we proceed with the proof of Lemma 2.2, we prove the following result which will be needed therein:

Lemma B.1. For $m=0,1, \ldots$, there is an $\varepsilon_{m}>0$ such that, for $0<\varepsilon \leq \varepsilon_{m}$, for $H=\mathcal{O}(\varepsilon)$, and for a general point $z=(x, y)$, the function $L_{m}$ is written as

$$
L_{m}(z)=\left(-\varepsilon^{-1} H\right)^{m+1}\left[\left(D_{y} g\right)_{0}(z)\right]^{m} g_{0}(z)+\mathcal{O}(\varepsilon)+\mathcal{O}\left(\left\|g_{0}(z)\right\|^{2}\right),
$$

where the notation " $(\cdot)_{0}(z)$ " stands for $(\cdot)(z, 0)$. The Jacobian $D_{y} L_{m}$ is written as

$$
\left(D_{y} L_{m}\right)(z)=\left(-\varepsilon^{-1} H\left(D_{y} g\right)_{0}\right)^{m+1}+\mathcal{O}(\varepsilon)+\mathcal{O}\left(\left\|g_{0}(z)\right\|^{2}\right) .
$$

Proof. For this proof, we write $(\cdot)_{0}$ instead of $(\cdot)_{0}(z)$ for the sake of brevity. The proof is by induction on $m$. For $m=0$, we recall equation (2.4),

$$
L_{0}=-\varepsilon^{-1} H g,
$$

and hence, expanding the smooth function $g$ in powers of $\varepsilon$, we find

$$
L_{0}=-\varepsilon^{-1} H g_{0}+\mathcal{O}(\varepsilon) \text {. }
$$

This is the desired formula for $L_{0}$. Differentiating both members of this formula with respect to $y$, we obtain

$$
D_{y} L_{0}=-\varepsilon^{-1} H\left(D_{y} g\right)_{0}+\mathcal{O}(\varepsilon) .
$$

This is the desired formula for $D_{y} L_{0}$. 
Next, we carry out the induction step for general $m$, namely we assume that

$$
\begin{aligned}
L_{m} & =\left(-\varepsilon^{-1} H\right)^{m+1}\left(D_{y} g\right)_{0}^{m} g_{0}+\mathcal{O}(\varepsilon)+\mathcal{O}\left(\left\|g_{0}(z)\right\|^{2}\right), \\
D_{y} L_{m} & =\left(-\varepsilon^{-1} H\left(D_{y} g\right)_{0}\right)^{m+1}+\mathcal{O}(\varepsilon)+\mathcal{O}\left(\left\|g_{0}(z)\right\|^{2}\right)
\end{aligned}
$$

and show that

$$
\begin{aligned}
L_{m+1} & =\left(-\varepsilon^{-1} H\right)^{m+2}\left(D_{y} g\right)_{0}^{m+1} g_{0}+\mathcal{O}(\varepsilon)+\mathcal{O}\left(\left\|g_{0}(z)\right\|^{2}\right), \\
D_{y} L_{m+1} & =\left(-\varepsilon^{-1} H\left(D_{y} g\right)_{0}\right)^{m+2}+\mathcal{O}(\varepsilon)+\mathcal{O}\left(\left\|g_{0}(z)\right\|^{2}\right) .
\end{aligned}
$$

By equation (2.9),

$$
L_{m+1}=-\varepsilon^{-1} H\left(D_{z} L_{m}\right) G=-\varepsilon^{-1} H\left[\varepsilon\left(D_{x} L_{m}\right) f+\left(D_{y} L_{m}\right) g\right] .
$$

Then, we substitute the induction hypothesis (B.5) into this expression. Application of the differential operator $(-H / \varepsilon)\left[\varepsilon\left(D_{x} \cdot\right) f+\left(D_{y} \cdot\right) g\right]$ on the smooth $\mathcal{O}(\varepsilon)+\mathcal{O}\left(\left\|g_{0}(z)\right\|^{2}\right)$ remainder does not alter its asymptotic magnitude. Moreover, the term $\varepsilon\left(D_{x} L_{m}\right) f$ is $\mathcal{O}(\varepsilon)$ and, hence, can be absorbed also in the remainder. Therefore, we are left with the term $(-H / \varepsilon)\left(D_{y} L_{m}\right) g$. Substituting $D_{y} L_{m}$ into this expression from the induction hypothesis (B.6), we arrive at the desired formula (B.7).

Finally, we prove the leading order formula (B.8). We differentiate both members of the leading order formula (B.7) with respect to $y$ and use the product rule derivative to evaluate the right member. The second term from the product rule is precisely the leading order term in equation (B.8). The other terms from the product rule,

$$
\left(-\varepsilon^{-1} H\right)^{m+2}\left[\sum_{r=1}^{m+1}\left(D_{y} g\right)_{0}^{m+1-r}\left(\left(D_{y}^{2} g\right)_{0}, g_{0}\right)\left(D_{y} g\right)_{0}^{r-1}\right]
$$

may be absorbed in the remainder since they all involve a factor that is linear in $g_{0}$. Thus, we have obtained the desired formula (B.8) and completed the proof of the lemma.

Proof of Lemma 2.2. We begin by estimating

$$
\left(\left(D_{z} L_{m}\right)\left(x, \tilde{h}_{m+1}(x), \varepsilon\right)\right) G\left(x, \tilde{h}_{m+1}(x), \varepsilon\right) .
$$

We may write

$$
\left(\left(D_{z} L_{m}\right)\left(\cdot, \tilde{h}_{m+1}(\cdot), \varepsilon\right)\right) G\left(\cdot, \tilde{h}_{m+1}(\cdot), \varepsilon\right)=\left[\left(D_{z} L_{m}\right)\left(\cdot, \tilde{h}_{m+1}(\cdot), \varepsilon\right)-\left(D_{z} L_{m}\right)\left(\cdot, h_{m}(\cdot), \varepsilon\right)\right] G\left(\cdot, \tilde{h}_{m+1}(\cdot), \varepsilon\right),
$$

since, by the definition of $\tilde{h}_{m+1}$,

$$
\left(\left(D_{z} L_{m}\right)\left(\cdot, h_{m}(\cdot), \varepsilon\right)\right) G\left(\cdot, \tilde{h}_{m+1}(\cdot), \varepsilon\right)=0 .
$$

Next, we have the following estimates of the asymptotic magnitudes of the two terms in the right member of equation (B.9):

$$
\tilde{h}_{m+1}=\sum_{i=0}^{m+1} \varepsilon^{i} h_{[i]}+\mathcal{O}\left(\varepsilon^{m+2}\right)
$$

by Lemma 2.1, and also

$$
h_{m}=\sum_{i=0}^{m} \varepsilon^{i} h_{[i]}+\mathcal{O}\left(\varepsilon^{m+1}\right)
$$


by the induction hypothesis. Thus,

$$
\tilde{h}_{m+1}-h_{m}=\mathcal{O}\left(\varepsilon^{m+1}\right),
$$

and hence Taylor's Theorem with remainder yields

$$
\left(D_{z} L_{m}\right)\left(\cdot, \tilde{h}_{m+1}(\cdot), \varepsilon\right)-\left(D_{z} L_{m}\right)\left(\cdot, h_{m}(\cdot), \varepsilon\right)=\mathcal{O}\left(\varepsilon^{m+1}\right),
$$

since $L_{m}$ and its derivatives are $\mathcal{O}(1)$. This is the desired estimate of the first term in the right member of equation (B.9).

It remains to estimate the second term, $G\left(\cdot, \tilde{h}_{m+1}(\cdot), \varepsilon\right)$ in the right member of equation (B.9). We recall that $G=\left(\begin{array}{c}\varepsilon f \\ g\end{array}\right)$, where $f$ and $g$ are $\mathcal{O}(1)$ in general. Hence, the first component of $G\left(\cdot, \tilde{h}_{m+1}(\cdot), \varepsilon\right)$ is plainly $\mathcal{O}(\varepsilon)$. The second component is as well, since Lemma 2.1 implies that $\tilde{h}_{m+1,0}=h_{[0]}$ and hence that $g\left(\cdot, \tilde{h}_{m+1}(\cdot), \varepsilon\right)=\mathcal{O}(\varepsilon)$, also. Therefore,

$$
G\left(\cdot, \tilde{h}_{m+1}(\cdot), \varepsilon\right)=\mathcal{O}(\varepsilon) .
$$

Combining the estimates (B.10) and (B.11), we see that the right member of equation (B.9) is $\mathcal{O}\left(\varepsilon^{m+2}\right)$, which leads to the desired result by an argument analogous to that in [2], Theorem 3.

Finally, the solution of the condition $L_{m+1}=0$ yields an $\mathrm{N}_{\mathrm{s}}$-dimensional manifold $\mathcal{L}_{m+1}$, as may be shown using the Implicit Function Theorem and [16], Theorem 1.13. It suffices to show that

$$
\operatorname{det}\left(D_{y} L_{m+1}\right)\left(\cdot, h_{m+1}(\cdot)\right) \neq 0 \text {. }
$$

Lemma B.1 yields a leading order formula for $D_{y} L_{m+1}$,

$$
\left(D_{y} L_{m+1}\right)(z)=\left(-\varepsilon^{-1} H\left(D_{y} g\right)_{0}\right)^{m+2}+\mathcal{O}(\varepsilon)+\mathcal{O}\left(\left\|g_{0}(z)\right\|\right) \text {. }
$$

Here, $z$ is a general point and $(\cdot)_{0}(z)=(\cdot)(z, 0)$. Next, we showed above that $h_{(m+1,0)}=h_{[0]}$. Recalling, then, equation (2.5), we obtain

$$
\left(D_{y} L_{m+1}\right)\left(x, h_{m+1}(x)\right)=\left[-\varepsilon^{-1} H\left(D_{y} g\right)_{0}\right]^{m+2}+\mathcal{O}(\varepsilon), \quad \text { for all } x \in K,
$$

where $\left(D_{y} g\right)_{0} \equiv\left(D_{y} g\right)\left(x, h_{[0]}(x), 0\right)$ here. Thus,

$$
\operatorname{det}\left(D_{y} L_{m+1}\right)\left(x, h_{m+1}(x)\right) \neq 0, \quad \text { for all } x \in K,
$$

by normal hyperbolicity and the proof is complete.

\section{REFERENCES}

[1] G. Browning and H.-O. Kreiss, Problems with different time scales for nonlinear partial differential equations. SIAM J. Appl. Math. 42 (1982) 704-718.

[2] J. Carr, Applications of Centre Manifold Theory, Applied Mathematical Sciences 35. Springer-Verlag, New York (1981).

[3] J. Curry, S.E. Haupt and M.E. Limber, Low-order modeling, initializations, and the slow manifold. Tellus 47A (1995) 145-161.

[4] N. Fenichel, Geometric singular perturbation theory for ordinary differential equations. J. Diff. Eq. 31 (1979) 53-98.

[5] C.W. Gear and I.G. Kevrekidis, Constraint-defined manifolds: a legacy-code approach to low-dimensional computation. J. Sci. Comp. 25 (2005) 17-28.

[6] C.W. Gear, T.J. Kaper, I.G. Kevrekidis and A. Zagaris, Projecting to a slow manifold: singularly perturbed systems and legacy codes. SIAM J. Appl. Dyn. Syst. 4 (2005) 711-732.

[7] S.S. Girimaji, Reduction of large dynamical systems by minimization of evolution rate. Phys. Rev. Lett. 82 (1999) $2282-2285$. 
[8] C.K.R.T. Jones, Geometric singular perturbation theory, in Dynamical Systems, Montecatini Terme, L. Arnold Ed., Lecture Notes Math. 1609, Springer-Verlag, Berlin (1994) 44-118.

[9] H.G. Kaper and T.J. Kaper, Asymptotic analysis of two reduction methods for systems of chemical reactions. Physica D 165 (2002) 66-93.

[10] C.T. Kelley, Iterative Methods for Linear and Nonlinear Equations, Frontiers In Applied Mathematics 16. SIAM Publications, Philadelphia (1995).

[11] I.G. Kevrekidis, C.W. Gear, J.M. Hyman, P.G. Kevrekidis, O. Runborg and C. Theodoropoulos, Equation-free, coarse-grained multiscale computation: enabling microscopic simulators to perform system-level analysis. Commun. Math. Sci. 1 (2003) $715-762$.

[12] H.-O. Kreiss, Problems with different time scales for ordinary differential equations. SIAM J. Numer. Anal. 16 (1979) $980-998$.

[13] H.-O. Kreiss, Problems with Different Time Scales, in Multiple Time Scales, J.H. Brackbill and B.I. Cohen Eds., Academic Press (1985) 29-57.

[14] E.N. Lorenz, Attractor sets and quasi-geostrophic equilibrium. J. Atmos. Sci. 37 (1980) 1685-1699.

[15] U. Maas and S.B. Pope, Simplifying chemical kinetics: intrinsic low-dimensional manifolds in composition space. Combust. Flame 88 (1992) 239-264.

[16] P.J. Olver, Applications of Lie Groups to Differential Equations, Graduate Texts in Mathematics 107. Springer-Verlag, New York (1986).

[17] G.M. Shroff and H.B. Keller, Stabilization of unstable procedures: A recursive projection method. SIAM J. Numer. Anal. 30 (1993) 1099-1120.

[18] P. van Leemput, W. Vanroose and D. Roose, Initialization of a Lattice Boltzmann Model with Constrained Runs. Report TW444, Catholic University of Leuven, Belgium (2005).

[19] P. van Leemput, C. Vandekerckhove, W. Vanroose and D. Roose, Accuracy of hybrid Lattice Boltzmann/Finite Difference schemes for reaction-diffusion systems. Multiscale Model. Sim. 6 (2007) 838-857.

[20] A. Zagaris, H.G. Kaper and T.J. Kaper, Analysis of the Computational Singular Perturbation reduction method for chemical kinetics. J. Nonlin. Sci. 14 (2004) 59-91.

[21] A. Zagaris, H.G. Kaper and T.J. Kaper, Fast and slow dynamics for the Computational Singular Perturbation method. Multiscale Model. Sim. 2 (2004) 613-638.

[22] A. Zagaris, C. Vandekerckhove, C.W. Gear, T.J. Kaper and I.G. Kevrekidis, Stability and stabilization of the constrained runs schemes for equation-free projection to a slow manifold. Numer. Math. (submitted). 